\title{
PLANAR LIMITS OF THREE-DIMENSIONAL INCOMPRESSIBLE FLOWS WITH HELICAL SYMMETRY
}

\author{
MILTON C. LOPES FILHO, ANNA L. MAZZUCATO, DONGJUAN NIU, \\ HELENA J. NUSSENZVEIG LOPES, AND EDRISS S. TITI
}

\begin{abstract}
Helical symmetry is invariance under a one-dimensional group of rigid motions generated by a simultaneous rotation around a fixed axis and translation along the same axis. The key parameter in helical symmetry is the step or pitch, the magnitude of the translation after rotating one full turn around the symmetry axis. In this article we study the limits of three-dimensional helical viscous and inviscid incompressible flows in an infinite circular pipe, with respectively no-slip and no-penetration boundary conditions, as the step approaches infinity. We show that, as the step becomes large, the three-dimensional helical flow approaches a planar flow, which is governed by the so-called two-and-half NavierStokes and Euler equations, respectively.
\end{abstract}

MSC Subject Classifications: 35Q35, 65M70.

Keywords: Helical symmetry, Navier-Stokes equations in thin domains.

August 21, 2018

\section{INTRODUCTION}

The helical groups are a family of one-dimensional subgroups of the rigid motions of three-dimensional Euclidean space consisting of simultaneous rotation around an axis and translation along the same axis, for which the ratio of angular rotation to translation is kept fixed. Each helical group is characterized by a parameter $\sigma \in \mathbb{R} \backslash\{0\}$, which we call the step or pitch, defined as the translation displacement along the symmetry axis after one full clockwise turn around the axis. The incompressible Navier-Stokes and Euler equations are covariant under the action of the helical group. Helically-symmetric or, simply, "helical" flows represent a physically interesting class of fluid motions, which interpolate between two-dimensional flows and axisymmetric flows, see for instance [2]. Indeed, the helical groups lie between rigid translations in one direction, associated with 2D flows, and rotation around a fixed axis, associated with axisymmetric flows. These regimes correspond to formally taking the limits $\sigma \rightarrow \infty$ and $\sigma \rightarrow 0$, respectively. The main goal of this work is to examine the precise nature of the limit $\sigma \rightarrow \infty$ for helical flows, in the case of viscous and inviscid incompressible flows in a circular pipe satisfying, respectively, no-slip and no-penetration boundary conditions. The limit $\sigma \rightarrow 0$ is more technical and, in some sense, less interesting, as we expect that helical flows will converge in the limit to axisymmetric, planar flows, a trivial 
special case of axisymmetric flows. In fact, periodicity in this case implies asymptotically high-frequency oscillations, with weak averaging in the vertical direction. The analysis of the limit $\sigma \rightarrow 0$ is closely related to that in some of the thin domain literature, particularly the special case referred to as $P D$, or periodic Dirichlet (see [8] for more details.) We reserve to study the limit $\sigma \rightarrow 0$ in future work.

We begin by recalling the known mathematical results concerning helical flows. As it is the case of two-dimensional flows and axisymmetric flows in cylindrical domains bounded away from the axis of symmetry, viscous incompressible helical flows are globally well posed. This result was proved by A. Mahalov, E. Titi and S. Leibovich in [12]. In fact, for the case of a circular pipe they established both global existence of a weak helical solution with initial data in $L^{2}$, and global existence and uniqueness of a strong solution with initial data in the Sobolev space $H^{1}$. (For a discussion about uniqueness of weak solutions, , within the class of all LerayHopf weak solutions of the three-dimensional Navier-Stokes with helical initial data, see [1].) The situation is different, and rather interesting, in the case of ideal fluid governed by the Euler equations, see [5,6]. As a matter of fact, an additional geometric condition is imposed on inviscid flows, akin to assuming no swirl in the axisymmetric setting, which we call no helical swirl or no helical stretching. Under this condition, B. Ettinger and E. Titi [6] showed global existence and uniqueness of weak solutions in an appropriate vorticity-stream function formulation. This formulation can be used, because, even for finite $\sigma$, the flow is essentially twodimensional, in the sense that it is completely determined by the dynamics of the first two components of the velocity field restricted to any cross section of the pipe.

The main result of this work is a convergence result of helical flows to certain flows, the dynamics of which is two dimensional. For this reason, we will call such limits planar flows, even though the velocity field can still have three nonzero components. More precisely, we show that, in the limit $\sigma \rightarrow \infty$, helical flows converge, respectively, to so-called 2 and $1 / 2$ dimensional flows in the viscous case, and to 2D Euler flows in the inviscid case. These results are established by first obtaining a set of symmetry-reduced equations equivalent to the original fluid equations, at least for regular flows. The unknowns in these equations are fields on a cross section of the pipe and, hence, depend on two spatial variables only. Convergence is then investigated via energy methods and compactness arguments. For the Navier-Stokes equations, energy estimates are sufficient to pass to the limit and give us a rate of convergence of order $1 / \sqrt{\sigma}$ in the energy norm.

One special difficulty in the viscous case is the way in which the divergence-free condition and the symmetry reduction interact when we vary $\sigma$. To be more precise, the symmetry reduction amounts to the fact that a helical vector field is entirely determined by its trace on a horizontal slice, say $D=\left\{x_{1}^{2}+x_{2}^{2}<1, x_{3}=0\right\}$, the trace being a three-component vector field in the plane. For a given $\sigma>0$ all threecomponent fields in $D$ may be extended in a unique way to helical vector fields in $D \times(0, \sigma)$. However, the resulting extension will not be divergence-free unless the original field in the slice satisfies a certain $\sigma$-dependent condition. In other words, after symmetry reduction, problems with different $\sigma$ reside in different function spaces, even if their physical domain $D$ is the same. This difficulty is bypassed 
in the inviscid case with the use of a stream function, under the "no helical swirl" condition.

The remainder of this article is divided into four sections. In Section 2 we fix notation and derive an equivalent formulation of helical symmetry for functions and vector fields. In Section 3, we perform the symmetry reduction on the NavierStokes equations. In Section 4 we study the limit $\sigma \rightarrow \infty$ for the viscous case, while in Section 5 we discuss the case of the Euler equations.

\section{PRELIMINARIES AND SYMMETRY REDUCTION}

We begin by recalling some standard notation for function spaces that will appear throughout the paper. If $\Omega$ is a domain in $\mathbb{R}^{d}$, we denote by $H^{k}(\Omega), k \in \mathbb{N}$, the standard $L^{2}$-based Sobolev spaces:

$$
H^{k}(\Omega)=\left\{f: \Omega \rightarrow \mathbb{R} ; f, \partial^{\alpha} f \in L^{2}(\Omega),|\alpha| \leq k\right\},
$$

where we employed the usual multiindex notation for derivatives, which are interpreted in the weak sense, while $W^{k, p}(\Omega)$ denotes $L^{p}$-based Sobolev spaces. By abuse of notation, if $\mathbf{u}: \Omega \rightarrow \mathbb{R}^{d}$ is a vector field, we will often write $\mathbf{u} \in H^{k}(\Omega)$ for $u \in\left(H^{k}(\Omega)\right)^{d}$, and we will drop the explicit dependence on the domain $\Omega$ when no confusion can arise. $H_{0}^{1}(\Omega)$ will denote the subspace of $H^{1}(\Omega)$ of functions with zero trace at the boundary $\partial \Omega$. If $\Omega$ is an unbounded domain, $L_{\mathrm{loc}}^{p}(\Omega)$ is the space of functions with $p$-th integrable power on each bounded open subset of $\Omega$. Lastly, we denote Hölder spaces by $C^{\alpha}(\Omega), \alpha \in \mathbb{R}_{+}$. Later in the paper, we will introduce other spaces adapted to the symmetry and geometry of the problem. Throughout, (, ) will denote the standard $L^{2}$ inner product.

One tool that will be used repeatedly in the analysis is the following interpolation inequality in two space dimensions, the so-called Ladyzhenskaya inequality. If $D$ is a smooth domain in $\mathbb{R}^{2}$ and $f \in H_{0}^{1}(D)$, then

$$
\|f\|_{L^{4}(D)}^{4} \leq 2\|f\|_{L^{2}(D)}^{2}\|\nabla f\|_{L^{2}(D)}^{2} .
$$

This inequality follows immediately from Lemma 1 on page 8 of [10].

Let $\Omega=\left\{x=\left(x_{1}, x_{2}, x_{3}\right) \in \mathbb{R}^{3} \mid x_{1}^{2}+x_{2}^{2} \leq 1\right\}=D \times \mathbb{R}$ be the infinite pipe with unit circular cross-section $D$ parallel to the $x_{3}$-axis.

We consider the initial-boundary-value problem for the incompressible NavierStokes (NSE) and Euler equations (EE) in $\Omega$. We recall the notion of helically symmetric solutions of these equations, studied in [6, 12].

We first give the definition of a helical vector field and a helical (scalar) function. We denote a point in $\mathbb{R}^{3}$ by $x=\left(x_{1}, x_{2}, x_{3}\right)$ in Cartesian coordinates. Given a nonzero number $\sigma \in \mathbb{R}$, we define the action of the helical group of transformations $G_{\sigma}$ on $\mathbb{R}^{3}$ by:

$$
S(\rho)(x)=\left(\begin{array}{c}
x_{1} \cos \rho+x_{2} \sin \rho \\
-x_{1} \sin \rho+x_{2} \cos \rho \\
x_{3}+\frac{\sigma}{2 \pi} \rho
\end{array}\right), \quad \rho \in \mathbb{R},
$$

that is, a rotation around the $x_{3}$ axis with simultaneous translation along the $x_{3}$ axis. $G_{\sigma}$ is uniquely determined by $\sigma$, which we will call the step (or pitch). 
Invariant curves for the action of the helical group $G_{\sigma}$ are helices having the $x_{3}$ axis as axis of symmetry. The cylinder $\Omega$ is an invariant set for the action of $G_{\sigma}$ for all $\sigma$. A change of sign in $\sigma$ corresponds to switching the orientation of the helices preserved by the group action from right-handed to left-handed. Without loss of generality, we will restrict our attention to the case of $\sigma>0$.

We will say that the smooth function $f(x)$ is helically symmetric, or simply helical, if $f$ is invariant under the action of $G_{\sigma}$, i.e., $f\left(S_{\rho} x\right)=f(x), \forall \rho \in$ $\mathbb{R}$. Similarly, we say that the smooth vector field $\mathbf{u}(x)$ is helically symmetric, or simply helical, if it is covariant with respect to the action of $G_{\sigma}$, i.e., $M(\rho) \mathbf{u}(x)=$ $\mathbf{u}(S(\rho) x)$ for all $\rho \in \mathbb{R}$, where

$$
M(\rho):=\left[\begin{array}{ccc}
\cos \rho & \sin \rho & 0 \\
-\sin \rho & \cos \rho & 0 \\
0 & 0 & 1
\end{array}\right] .
$$

We find it convenient to give an alternative definition of helical symmetry as follows. We re-write a vector field $\mathbf{u}(x)=\left(u^{1}, u^{2}, u^{3}\right)\left(x_{1}, x_{2}, x_{3}\right)$ with respect to the moving orthonormal frame associated to standard cylindrical coordinates $(r, \theta, z)$,

$$
\mathbf{e}_{\mathbf{r}}=(\cos \theta, \sin \theta, 0), \quad \mathbf{e}_{\theta}=(-\sin \theta, \cos \theta, 0), \quad \mathbf{e}_{\mathbf{z}}=(0,0,1),
$$

as:

$$
\mathbf{u}=u_{r} \mathbf{e}_{\mathbf{r}}+u_{\theta} \mathbf{e}_{\theta}+u_{z} \mathbf{e}_{\mathbf{z}}
$$

where $u_{r}, u_{\theta}, u_{z}$ are functions of $(r, \theta, z)$. We introduce two new independent variables in place of $\theta$ and $z$ :

$$
\eta:=\frac{\sigma}{2 \pi} \theta+z, \xi:=\frac{\sigma}{2 \pi} \theta-z .
$$

As shown in [6] for instance, a (smooth) function $p=p(r, \theta, z)$ is a helical function if and only if, when expressed in the $(r, \xi, \eta)$ variables, it is independent of $\xi: p=$ $q\left(r, \frac{\sigma}{2 \pi} \theta+z\right)$, for some $q=q(r, \eta)$ Similarly, a (smooth) vector field $\mathbf{u}$ is helical if and only if there exist $v_{r}, v_{\theta}, v_{z}$, functions of $(r, \eta)$ such that $u_{r}=v_{r}\left(r, \frac{\sigma}{2 \pi} \theta+z\right)$, $u_{\theta}=v_{\theta}\left(r, \frac{\sigma}{2 \pi} \theta+z\right), u_{z}=v_{z}\left(r, \frac{\sigma}{2 \pi} \theta+z\right)$.

We note that a vector field $\mathbf{u}$ is invariant under the action of $G_{\sigma}$ for all $\sigma \neq 0$ if and only if $v_{r}, v_{\theta}, v_{z}$ are functions of $r$ only. In particular, planar, circularly symmetric flows, that is flows for which $v_{r}=v_{z} \equiv 0$ and $v_{\theta}$ is a radial function, are a (very) special case of helical flows.

The change of variables $\left(x_{1}, x_{2}, x_{3}\right) \mapsto(r, \xi, \eta)$ introduced above has often been used to characterize helical symmetry, and, in fact, it does provide a simple, geometrically elegant description of invariance for both scalar functions and vector fields. However, to obtain estimates on solutions of the fluid equations, we find that an alternative characterization actually simplifies calculations, by avoiding moving frames. As a matter of fact, we show in the following proposition that sufficiently smooth functions and fields with helical symmetry are essentially two dimensional, 
in the sense that they are uniquely determined by their trace on any "slice" $\Omega \cap\{z=$ constant \}, which can be canonically identified with the unit disk $D \subset \mathbb{R}^{2}$.

Below we will make use of the following notation, where we employ Cartesian coordinates and frames. Given $y=\left(y_{1}, y_{2}\right)$ we let $y^{\perp}=\left(-y_{2}, y_{1}\right)$ and we set

$$
E \equiv y^{\perp} \cdot \nabla_{y} \text {. }
$$

We also use the notation $\mathbf{V}_{H}=\left(V^{1}, V^{2}, 0\right)$ for the horizontal component of the vector $\mathbf{V}=\left(V^{1}, V^{2}, V^{3}\right)$, and we denote the vector $\left(-V^{2}, V^{1}, 0\right)$ by $\mathbf{V}_{H}^{\perp}$.

Proposition 2.1. Let $\mathbf{u}=\mathbf{u}(x)$ be a smooth helical vector field and let $p=p(x)$ be a smooth helical function, where $x=\left(x_{1}, x_{2}, x_{3}\right)$. Then there exist unique $\mathbf{w}=\left(w^{1}, w^{2}, w^{3}\right)=\left(w^{1}, w^{2}, w^{3}\right)\left(y_{1}, y_{2}\right)$ and $q=q\left(y_{1}, y_{2}\right)$ such that

$$
\mathbf{u}(x)=M\left(2 \pi x_{3} / \sigma\right) \mathbf{w}(y(x)), \quad p=p(x)=q(y(x)),
$$

with $M(\rho)$ given in 2.2), and

$$
y(x)=\left[\begin{array}{c}
y_{1} \\
y_{2}
\end{array}\right]=\left[\begin{array}{cc}
\cos \left(2 \pi x_{3} / \sigma\right) & -\sin \left(2 \pi x_{3} / \sigma\right) \\
\sin \left(2 \pi x_{3} / \sigma\right) & \cos \left(2 \pi x_{3} / \sigma\right)
\end{array}\right]\left[\begin{array}{c}
x_{1} \\
x_{2}
\end{array}\right] .
$$

Conversely, if $\mathbf{u}$ and $p$ are defined through (2.5) for some $\mathbf{w}=\mathbf{w}\left(y_{1}, y_{2}\right), q=$ $q\left(y_{1}, y_{2}\right)$, then $\mathbf{u}$ is a helical vector field and $p$ is a helical scalar function.

We omit the proof, which is a standard application of vector calculus.

In what follows, for notational convenience we set

$$
m^{\sigma}\left(x_{3}\right)=\left[\begin{array}{cc}
\cos \left(2 \pi x_{3} / \sigma\right) & -\sin \left(2 \pi x_{3} / \sigma\right) \\
\sin \left(2 \pi x_{3} / \sigma\right) & \cos \left(2 \pi x_{3} / \sigma\right)
\end{array}\right],
$$

so that

and

$$
y\left(x_{1}, x_{2}, x_{3}\right)=\left[\begin{array}{l}
y_{1} \\
y_{2}
\end{array}\right]=m^{\sigma}\left(x_{3}\right)\left[\begin{array}{l}
x_{1} \\
x_{2}
\end{array}\right],
$$

$$
M^{\sigma}\left(x_{3}\right) \equiv M\left(2 \pi x_{3} / \sigma\right)=\left[\begin{array}{cc}
\left(m^{\sigma}\left(x_{3}\right)\right)^{T} & 0 \\
0 & 1
\end{array}\right] .
$$

It is clear, from Proposition (2.1) above that any smooth helical flow is periodic in $x_{3}$, both velocity and pressure, with period the step $\sigma$. We can therefore state the initial-boundary-value problem for the Navier-Stokes equations in the fundamental domain $\Omega^{\sigma}:=D \times(0, \sigma)$ :

$$
\begin{cases}\partial_{t} \mathbf{u}+(\mathbf{u} \cdot \nabla) \mathbf{u}=-\nabla p+\nu \Delta \mathbf{u}+\mathbf{f}, & \text { in }(0,+\infty) \times \Omega^{\sigma} ; \\ \operatorname{div} \mathbf{u}=0, & \text { in }[0,+\infty) \times \Omega^{\sigma} ; \\ \mathbf{u}\left(t, x^{\prime}, x_{3}\right)=0, & \text { for } t \in[0,+\infty), \quad\left|x^{\prime}\right|=1,0 \leq x_{3} \leq \sigma \\ \mathbf{u}\left(t, x^{\prime}, x_{3}\right)=\mathbf{u}\left(t, x^{\prime}, x_{3}+\sigma\right) & \text { for } t \in[0,+\infty), \quad x^{\prime} \in D ; \\ p\left(t, x^{\prime}, x_{3}\right)=p\left(t, x^{\prime}, x_{3}+\sigma\right) & \text { for } t \in[0,+\infty), \quad x^{\prime} \in D ; \\ \mathbf{u}(0, x)=\mathbf{u}_{0}, & x \in \Omega^{\sigma},\end{cases}
$$

where we set $x^{\prime}=\left(x_{1}, x_{2}\right)$, so that $x=\left(x^{\prime}, x_{3}\right)$. 
The Euler equations are formally obtained by setting $\nu=0$ above and by replacing the no-slip boundary condition $\left.\mathbf{u}\right|_{\partial \Omega^{\sigma}}=0$ with the no-penetration condition $\mathbf{u} \cdot x^{\prime}=0$ on $\partial \Omega^{\sigma}$. We discuss Euler solutions in Section 5.

In what follows, for simplicity we set any body forcing $\mathbf{f} \equiv 0$, and take the viscosity coefficient $\nu=1$, as we do not contend ourselves with the vanishing viscosity limit in this work. We plan to study the interplay between the limits $\nu \rightarrow 0$ and $\sigma \rightarrow \infty$ in future work.

We denote by $C_{p e r}^{\alpha}\left(\overline{\Omega^{\sigma}}\right)$ the subspace of $C^{\alpha}(\bar{\Omega}), \alpha \in \mathbb{R}_{+}$of functions that are $\sigma$-periodic in $x_{3}$, and by $C_{c, p e r}^{\infty}\left(\Omega^{\sigma}\right)$ the space of functions which are $\sigma$-periodic in $x_{3}$ and compactly supported in $D$ for each fixed $x_{3} \in[0, \sigma]$. We also denote by $H_{0, p e r}^{1}\left(\Omega^{\sigma}\right)$ the closure of $C_{c, p e r}^{\infty}\left(\Omega^{\sigma}\right)$ in $H^{1}\left(\Omega^{\sigma}\right)$, and by $H_{\text {per }}^{-1}\left(\Omega^{\sigma}\right)$ its dual. We note that the closure of the subspace of $C_{c, p e r}^{\infty}\left(\Omega^{\sigma}\right)$ of divergence-free vector field is the subspace $\left\{\mathbf{u} \in H_{0, p e r}^{1}\left(\Omega^{\sigma}\right) \mid \operatorname{div} u=0\right\}$, where derivatives are taken in the weak sense.

In the remainder of the paper we will consider solutions to 2.7 and the corresponding inviscid system (5.1) with initial data $u_{0}$ of limited regularity. More specifically, $u_{0}$ will be taken in $H_{0, p e r}^{1}\left(\Omega^{\sigma}\right)$ for Navier-Stokes and in $H_{p e r}^{1}\left(\Omega^{\sigma}\right)$ with initial vorticity curl $u_{0} \in L^{\infty}\left(\Omega^{\sigma}\right)$ for Euler. We now briefly discuss helical symmetry in this context.

Definition 2.1. Let $p \in H_{p e r}^{1}\left(\Omega^{\sigma}\right)$. We say that $p$ has helical symmetry if there exists a sequence of smooth, helical functions $p_{n}$ such that $p=\lim _{n \rightarrow \infty} p_{n}$ in $H_{p e r}^{1}\left(\Omega^{\sigma}\right)$. Similarly, we say that a vector field $\mathbf{u}$ in $H_{p e r}^{1}\left(\Omega^{\sigma}\right)^{3}$ has helical symmetry if $\mathbf{u}$ is a strong limit in $H_{\text {per }}^{1}\left(\Omega^{\sigma}\right)^{3}$ of a sequence of smooth, helical vector fields $\mathbf{u}_{n}$.

We next show that the characterization of helical symmetry given in Proposition 2.1 carries over to functions and vector fields in $H^{1}$.

Proposition 2.2. Let $\mathbf{u} \in\left(H_{p e r}^{1}\left(\Omega^{\sigma}\right)\right)^{3}, p \in H_{p e r}^{1}\left(\Omega^{\sigma}\right)$ be, respectively, a helical vector field and a helical function. Then, there exist a unique $\mathrm{w} \in H^{1}(D)^{3}$ and $q \in H^{1}(D)$, where $D$ is the unit disk in $\mathbb{R}^{2}$, such that

$$
\begin{aligned}
& \mathbf{u}(x)=M^{\sigma}\left(x_{3}\right) \mathbf{w}\left(m^{\sigma}\left(x_{3}\right) x^{\prime}\right), \\
& \left.p(x)=q\left(m^{\sigma}\left(x_{3}\right) x^{\prime}\right)\right),
\end{aligned}
$$

Conversely, given $\mathbf{w} \in H^{1}(D)^{3}$ and $q \in H^{1}(D)$, if $\mathbf{u}$ and $p$ are defined through (2.8), then $\mathbf{u} \in\left(H_{p e r}^{1}\left(\Omega^{\sigma}\right)\right)^{3}, p \in\left(H_{p e r}^{1}\left(\Omega^{\sigma}\right)\right)$, and they have helical symmetry.

Proof. We only consider the case of a helical vector field $\mathbf{u}$. The case of a helical function is similar and simpler. By definition, there exist helical vector fields $\mathbf{u}_{n} \in$ $C^{\infty}\left(\bar{\Omega}^{\sigma}\right)$ such that $u_{n} \rightarrow u$ strongly in $H_{p e r}^{1}\left(\Omega^{\sigma}\right)$. By Proposition 2.1 , for each $\mathbf{u}_{n}$ there exists a unique, smooth $\mathbf{w}_{n}$ such that $\mathbf{w}_{n}\left(x^{\prime}\right)=\left(M^{\sigma}\left(x_{3}\right)\right)^{T} \mathbf{u}_{n}\left(\left(m^{\sigma}\left(x_{3}\right)\right)^{T} x^{\prime}\right)$, for all $x^{\prime}=\left(x_{1}, x_{2}\right) \in D$. Therefore, the expression on the right-hand side is independent of $x_{3}$ and $\nabla_{x}\left(M^{\sigma}\left(x_{3}\right)\right)^{T} \mathbf{u}\left(\left(m^{\sigma}\left(x_{3}\right)\right)^{T} x^{\prime}\right)=\left(\nabla_{x^{\prime}} \mathbf{w}\left(x^{\prime}\right), 0\right)$. If we define

$$
\mathbf{w}\left(x^{\prime}, x_{3}\right):=\left(M^{\sigma}\left(x_{3}\right)\right)^{T} \mathbf{u}\left(\left(m^{\sigma}\left(x_{3}\right)\right)^{T} x^{\prime}\right),
$$


then $\partial_{x_{3}} \mathbf{w}\left(x^{\prime}, x_{3}\right)=0$ in weak sense, since it is true for $\mathbf{w}_{n}$ and $\nabla_{x} \mathbf{u}_{n} \rightarrow \nabla \mathbf{u}$ strongly in $L^{2}\left(\Omega^{\sigma}\right)$. Consequently, $\mathbf{w}$ is independent of $x_{3}$ for almost all $x^{\prime} \in$ $D$ (functions with vanishing weak derivatives are constant, see e.g. [11, Theorem 6.11]) and $\mathbf{w} \in H^{1}(D)$. Furthermore,

$$
\left\|\mathbf{w}_{n}-\mathbf{w}\right\|_{H^{1}(D)} \leq C \sqrt{\sigma}\left\|\mathbf{u}_{n}-\mathbf{u}\right\|_{H^{1}\left(\Omega_{\sigma}\right)},
$$

by a simple change of variables, so that $\mathbf{w}_{n} \rightarrow \mathbf{w}$ strongly in $H^{1}(D)$. The converse statement is a direct consequence of (2.8).

Remark 2.1. The proof of Proposition 2.2 shows that if $\mathbf{u} \in H^{m}\left(\Omega^{\sigma}\right), m \in \mathbb{N}$, then $\mathbf{w} \in H^{m}(D)$ and the $H^{m}$ norm of $\mathbf{w}$ on $D$ is bounded by the $H^{m}$ norm of $\mathbf{u}$ on $\Omega^{\sigma}$ with constants that depend on $\sigma$. The same result holds in $L^{p}$-Sobolev spaces $W_{p e r}^{m, p}\left(\Omega^{\sigma}\right)$ for $1 \leq p<\infty$. These spaces are defined in a manner totally analogous to $H_{p e r}^{m}\left(\Omega^{\sigma}\right)$.

We next recall the notion of weak and strong Navier-Stokes solutions. By a classical solution of (2.7) on the time interval $[0, T]$, we mean a vector field $\mathbf{u} \in$ $C^{1}\left([0, T] ; C^{2}\left(\overline{\Omega^{\sigma}}\right)\right.$, together with a function $p \in C^{1}\left([0, T), C^{1}\left(\Omega_{\sigma}\right)\right)$ such that the equations, and the initial and boundary conditions are met pointwise in $t$ and $x$. By a weak solution on the time interval $[0, T)$, we mean a divergence-free vector field $\mathbf{u}:[0, T) \times \Omega^{\sigma} \rightarrow \mathbb{R}^{3}$ such that $\mathbf{u} \in C_{w}\left([0, T) ; L^{2}\left(\Omega^{\sigma}\right)\right) \cap L^{2}\left((0, T) ; H_{0, p e r}^{1}\left(\Omega^{\sigma}\right)\right)$ and $\partial_{t} \mathbf{u} \in L^{1}\left((0, T), H_{\text {per }}^{-1}\left(\Omega^{\sigma}\right)\right)$, satisfying the equations in the sense of distributions and the initial condition $\mathbf{u}(0)=\mathbf{u}_{0} \in L^{2}\left(\Omega^{\sigma}\right)$. Here, $C_{w}\left([0, T) ; L^{2}\right)$ is the space of all functions of $t$ with values in $L^{2}$ that are continuous w.r.t. the weak topology on $L^{2}$. We remark that weak solutions satisfy the Dirichlet (noslip) boundary conditions at least in trace sense on the boundary for almost all $0<t<T$. By a strong solution we mean a weak solution that satisfies in addition $u \in L^{\infty}\left([0, T) ; H_{0, p e r}^{1}\left(\Omega^{\sigma}\right)\right) \cap L^{2}\left((0, T) ; H_{p e r}^{2}\left(\Omega^{\sigma}\right) \cap H_{0, p e r}^{1}\left(\Omega^{\sigma}\right)\right)$ and the condition $\mathbf{u}_{0} \in H_{0, p e r}^{1}\left(\Omega^{\sigma}\right)$. It then follows that there exists an associated pressure function $p \in L^{2}\left((0, T) ; H^{1}\left(\Omega^{\sigma}\right)\right)$. A strong helical solution will denote a strong solution that is a helical field in the sense of Definition 2.1. We recall that any strong solution of the Navier-Stokes equations is unique and smooth for $t>0$ (see e.g., [14, Theorem 1.8.2]). Hence, strong solutions are actually classical solutions on any time interval $[\delta, T], \delta>0$. It was shown in [12, Theorem 3.4] that weak solutions of 2.7) with helical symmetry are unique, global in time, and agree with a strong solution, if the initial data belongs to $H_{0, p e r}^{1}\left(\Omega^{\sigma}\right)$ and the associated pressure $p$ is also a helical function. (See also [1] for more elaborate discussion regarding this matter.)

\section{SyMmETRY REDUCTION FOR THE NAVIER-STOKES EQUATIONS}

In this section we derive a set of symmetry-reduced equations that completely capture the dynamics of the original system under the hypothesis of helical symmetry.

We begin by deriving the symmetry-reduced system under the hypothesis that $(\mathbf{u}, p)$ are classical solutions of (2.7) and have helical symmetry. Let $\mathbf{w}=\mathbf{w}\left(t, y_{1}, y_{2}\right)$ 
be given in terms of $\mathbf{u}$ by Proposition 2.5. We will derive from Navier-Stokes the equations satisfied by $\mathbf{w}$. Smoothness of $\mathbf{u}$ and $\mathbf{w}$ justifies all the algebraic manipulations. For ease of notation, in this proof we write $M^{T}$ for $\left[\left(M^{\sigma}\right)\left(x_{3}\right)\right]^{T}$. We multiply the momentum equation in 2.7) by $M^{T}$ and identify each term in the resulting expression as follows to obtain:

$$
\begin{aligned}
& M^{T} \partial_{t} \mathbf{u}=\partial_{t} \mathbf{w}, \\
& M^{T}\left[\left(\mathbf{u} \cdot \nabla_{x}\right) \mathbf{u}\right]=\left(\mathbf{w}_{H} \cdot \nabla_{y}\right) \mathbf{w}+\left(\frac{2 \pi}{\sigma}\right) w^{3} E \mathbf{w}-\left(\frac{2 \pi}{\sigma}\right) w^{3} \mathbf{w}_{H}^{\perp}, \\
& M^{T} \nabla_{x} p=\left(\nabla_{y} q\right)_{H}+\left(\frac{2 \pi}{\sigma}\right) E q \mathbf{e}_{3}, \\
& M^{T} \Delta_{x} \mathbf{u}=\Delta_{y} \mathbf{w}+\left(\frac{2 \pi^{2}}{\sigma^{2}}\right)\left[E^{2} \mathbf{w}-2 E \mathbf{w}_{H}^{\perp}-\mathbf{w}_{H}\right],
\end{aligned}
$$

where $E$ is the operator defined in (2.4). We similarly perform the symmetry reduction on the incompressibility condition for $\mathbf{u}$ to obtain

$$
\operatorname{div}_{x} \mathbf{u}=\operatorname{div}_{y} \mathbf{w}_{H}+\left(\frac{2 \pi}{\sigma}\right) E w^{3} .
$$

Therefore, we find that $\mathrm{w}$ and $q$ satisfy the following initial-boundary-value problem:

$$
\begin{aligned}
& \partial_{t} \mathbf{w}+\left(\mathbf{w}_{H} \cdot \nabla_{y}\right) \mathbf{w}+\frac{2 \pi}{\sigma} w^{3}\left[E \mathbf{w}-\mathbf{w}_{H}^{\perp}\right]=-\left(\nabla_{y} q\right)_{H} \\
& -\frac{2 \pi}{\sigma} E q \mathbf{e}_{3}+\Delta_{y} \mathbf{w}+\frac{4 \pi^{2}}{\sigma^{2}}\left[E^{2} \mathbf{w}-2 E \mathbf{w}_{H}^{\perp}-\mathbf{w}_{H}\right], \\
& \operatorname{div}_{y} \mathbf{w}_{H}+\frac{2 \pi}{\sigma} E w^{3}=0, \quad t>0, \quad y \in D, \\
& \mathbf{w}(t, y)=0, \quad t>0, \quad|y|=1, \\
& \mathbf{w}(0, y)=\mathbf{w}_{0}(y), \quad y \in D,
\end{aligned}
$$

where $\mathbf{w}_{0}$ is related to $\mathbf{u}_{0}$ via (2.5).

Before giving a weak formulation of the above initial-boundary-value problem, we note that the operator $E=y^{\perp} \cdot \nabla_{y}$ is anti-selfadjoint, i.e., $E^{*}=-E$, since $\operatorname{div}_{y} y^{\perp}=0$. If we write (3.3b) as $A \mathbf{w}=0$, for some matrix operator $A$ with w a column vector, it follows that $A$ and its adjoint $A^{*}$ are given by:

$$
A:=\left[\begin{array}{lll}
\partial_{y_{1}}, & \partial_{y_{2}}, & \frac{2 \pi}{\sigma} E
\end{array}\right], \quad A^{*}:=\left[\begin{array}{c}
-\partial_{y_{1}} \\
-\partial_{y_{2}} \\
-\frac{2 \pi}{\sigma} E
\end{array}\right] .
$$

It can be easily checked that the (scalar) second-order operator $A A^{*}=-\Delta_{y}-$ $\frac{4 \pi^{2}}{\sigma^{2}} E^{2}$ is elliptic for any $\sigma \neq 0$.

We will call a vector field $\mathbf{w}$ on $[0, T) \times D$ a weak solution of (3.3) if $\mathbf{w} \in$ $C_{w}\left([0, T) ; L^{2}(D)\right) \cap L^{2}\left((0, T) ; H_{0}^{1}(D)\right), \partial_{t} \mathbf{w} \in L^{1}\left((0, T) ; H^{-1}(D), \mathbf{w}(0)=\right.$ $\mathbf{w}_{0} \in L^{2}(D)$, w satisfies the constraint (3.3b) in th sense of distributions, and for all (vector-valued) test functions $\boldsymbol{\Phi} \in C_{c}^{\infty}([0, T) \times D)$ that satisfy $3.3 \mathrm{~b}$, 


$$
\begin{array}{r}
\int_{0}^{t} \int_{D} \mathbf{w} \cdot \partial_{t} \boldsymbol{\Phi} d y d t+\frac{2 \pi}{\sigma} \int_{0}^{t} \int_{D} w^{3}\left(\mathbf{\Phi} \cdot \mathbf{w}_{H}^{\perp}+E \boldsymbol{\Phi} \cdot \mathbf{w}\right) d y d t+ \\
\int_{0}^{t} \int_{D} \Delta \boldsymbol{\Phi} \cdot \mathbf{w} d y d t+\frac{4 \pi^{2}}{\sigma^{2}} \int_{0}^{t} \int_{D}\left(E^{2} \mathbf{\Phi} \cdot \mathbf{w}+2 E \boldsymbol{\Phi} \cdot \mathbf{w}_{H}^{\perp}\right) d y d t \\
-\frac{4 \pi^{2}}{\sigma^{2}} \int_{0}^{t} \int_{D} \mathbf{\Phi} \cdot \mathbf{w}_{H} d y d t=\int_{D} \mathbf{\Phi}(0) \cdot \mathbf{w}(0) d y
\end{array}
$$

A weak solution will be called a strong solution if, in addition, $\mathbf{w} \in L^{\infty}\left([0, T) ; H_{0}^{1}(D)\right)$ $\cap L^{2}\left((0, T) ; H^{2}(D) \cap H_{0}^{1}(D)\right)$ and $\mathbf{u}_{0} \in H_{0}^{1}(D)$. By interpolation then, w $\in$ $C\left((0, T) ; H_{0}^{1}(D)\right)$ (c.f. e.g. [15, Lemma 4.8 p. 570]). By projecting the momentum equation (3.3a) onto the kernel of the operator $A$, one obtains an elliptic equation for the pressure $q$ :

$$
A A^{*} q=A\left[\left(\mathbf{w}_{H} \cdot \nabla_{y}\right) \mathbf{w}-\frac{2 \pi}{\sigma} w^{3}\left(E \mathbf{w}-\mathbf{w}_{H}^{\perp}\right)-\frac{4 \pi^{2}}{\sigma^{2}}\left(2 E \mathbf{w}_{H}^{\perp}+\mathbf{w}_{H}\right)\right],
$$

and by elliptic regularity, it follows that $q \in L^{1}\left([0, T) ; H^{1}(D)\right)$.

In the following proposition we establish the relationship between strong solutions to the Naviers-Stokes system 2.7) and strong solutions of the symmetryreduced system (3.3).

Proposition 3.1. Let $\mathbf{u}_{0} \in H_{0, p e r}^{1}\left(\Omega^{\sigma}\right)$ be a divergence-free, helical vector field. Let $\mathbf{u}$ be the unique, strong helical solution of (2.7) on $[0, T)$, for any $T>0$, with initial condition $\mathbf{u}_{0}$ and associated pressure function $p$. Then, the vector function $\mathbf{w}=\left(w^{1}, w^{2}, w^{3}\right)$ and scalar function $q$, defined through (2.8) from $\mathbf{u}$ and $p$, give a strong solution of the reduced system (3.3).

Conversely, let $\mathbf{w}$ be a strong solution of (3.3) and associated pressure q. Then, if $\mathbf{u}$ and $p$ are defined from $\mathbf{w}$ and $q$ via (2.8), $\mathbf{u}$ is a strong helical solution of (2.7). In particular, strong solutions of (3.3) are unique.

Proof. By Definition 2.1, there exists a sequence of smooth, helical functions $\mathbf{u}_{0, n}$ on $\Omega^{\sigma}$ such that $\mathbf{u}_{0, n} \rightarrow \mathbf{u}_{0}$ strongly in $H_{0, p e r}^{1}\left(\Omega^{\sigma}\right)$. Let $\mathbf{u}_{n}$ be the unique, classical helical solution of (3.3) with initial data $\mathbf{u}_{0, n}$, and pressure $p_{n}$. The sequence $\left\{\mathbf{u}_{n}\right\}$ is uniformly bounded in $L^{\infty}\left([0, T) ; H_{0, p e r}^{1}\left(\Omega^{\sigma}\right)\right) \cap L^{2}\left((0, T) ; H_{p e r}^{2}\left(\Omega^{\sigma}\right) \cap\right.$ $\left.H_{0, p e r}^{1}\left(\Omega^{\sigma}\right)\right)$ and $\left\{\partial_{t} \mathbf{u}_{n}\right\}$ is uniformly bounded in $L^{1}\left([0, T) ; H_{p e r}^{-1}\left(\Omega^{\sigma}\right)\right)$. Therefore, by interpolation and Rellich's theorem, there exists a subsequence converging strongly in $H^{-\epsilon}\left([0, T) ; H_{0, p e r}^{1}\left(\Omega^{\sigma}\right)\right) \cap L^{2}\left((0, T) ; H_{p e r}^{1-\epsilon}\left(\Omega^{\sigma}\right)\right)$, for all $\epsilon>0$, weakly in $L^{2}\left((0, T) ; H^{2}\left(\Omega^{\sigma}\right)\right)$, and weakly-* in $L^{\infty}\left([0, T) ; H_{0, p e r}^{1}\left(\Omega^{\sigma}\right)\right)$, such that $\partial_{t} \mathbf{u}_{n}$ converges weakly in $L^{1}\left((0, T) ; H^{-1}(D)\right)$. The limit $\mathbf{u}$ is then a weak solution of (3.3) with initial data $\mathbf{u}_{0}$ (by arguments similar to those showing existence of Leray-Hopf weak solutions, cf. [15, Theorem 5.9, Chap. 17]. )

Since weak solutions agree with strong solutions as long as the latter exists, we must have that $\mathbf{u}$ is the unique, strong helical solution of (3.3) with initial data $\mathbf{u}_{0}$. Hence, the whole sequence $\left\{\mathbf{u}_{m}\right\}$ converges to $\mathbf{u}$ by uniqueness of the limit. A similar argument gives convergence of $p_{n}$ to $p$ in $L^{1}\left((0, T) ; H^{1}(D)\right)$. 
Let now $\mathbf{w}_{n}$ be associated to $\mathbf{u}_{n}$ by (2.5). Then, $\mathbf{w}_{n}$ is a classical solution of (3.3), with associated pressure $q_{n}$ given by (2.5) in terms of $p_{n}$, by the calculations at the beginning of this section. Furthermore, the proof of Proposition 2.1 implies that all Sobolev norms of $\mathbf{w}_{n}$ and $q_{n}$ are bounded by the corresponding Sobolev norms of $\mathbf{u}_{n}$ with constants depending on $\sigma$. Hence, the sequence $\left\{\mathbf{w}_{n}\right\}$ is uniformly bounded in $L^{\infty}\left([0, T) ; H_{0}^{1}(D)\right) \cap L^{2}\left((0, T) ; H^{2}(D) \cap H_{0}^{1}(D)\right)$. From the equations, it follows that $\partial_{t} \mathbf{w}_{n}$ is uniformly bounded in $L^{1}\left((0, T) ; H^{-1}(D)\right)$. Hence, by interpolation and Rellich's theorem there exists a subsequence converging strongly in $H^{-\epsilon}\left([0, T) ; H_{0}^{1}(D)\right) \cap L^{2}\left((0, T) ; H^{1-\epsilon}(D)\right)$, for all $\epsilon>0$, weakly in $L^{2}\left((0, T) ; H^{2}(D)\right)$, and weakly-* in $L^{\infty}\left([0, T) ; H_{0}^{1}(D)\right)$ to a weak solution $\mathbf{w}$ of the symmetry-reduced system 3.3 . Since $\mathbf{w} \in L^{\infty}\left([0, T) ; H_{0}^{1}(D)\right) \cap$ $L^{2}\left((0, T) ; H^{2}(D) \cap H_{0}^{1}(D)\right)$, w is a strong solution of the reduced system. Also, by refining the subsequence if needed, we can assume that $\left\{q_{n}\right\}$ converges weakly in $L^{1}\left((0, T) ; H^{1}(D)\right)$. Furthermore, the convergence of $\mathbf{u}_{n}$ to $\mathbf{u}$ implies weak convergence of the right-hand side of (3.5) in $\left.L^{1}(0, T) ; H^{-1}(D)\right)$ and, hence, $q$ is a weak solution of the pressure equation. Lastly, since $\mathbf{w}$ and $q$ in 2.8 are unique, given $\mathbf{u}$ and $p$, these must agree with the limits of $\mathbf{u}_{n}$ and $p_{n}$. The first half of the theorem is established.

The converse follows by similar arguments, using again the uniqueness in the relation between $\mathbf{u}, p$ with $\mathbf{w}, q$ of Proposition 2.2. Energy estimates for strong solutions of the symmetry-reduced equations are given in Propositions 4.2 and 4.3. Uniqueness of strong solutions to the reduced equations then follows from uniqueness of helical, strong solutions of the Navier-Stokes equations.

\section{THE LIMIT $\sigma \rightarrow \infty$ FOR THE NAVIER-STOKES SYSTEM}

The purpose of this section is to discuss the limit $\sigma \rightarrow \infty$ for helical solutions of the Navier-Stokes equations. To emphasize the dependence of the solution on the parameter $\sigma$, we will write $\mathbf{u}^{\sigma}$ and $p^{\sigma}$ for $\mathbf{u}$ and $p$.

Next, we recall that to any helical vector field $\mathbf{u}^{\sigma}$ in $H^{1}\left(\Omega^{\sigma}\right)$ we can associate a three-component vector function $\mathbf{w}^{\sigma}$ in $H^{1}(D)$ by means of Proposition 2.2 The divergence-free condition on $\mathbf{u}^{\sigma}$ is recast as $3.3 \mathrm{~b}$ ) for $\mathbf{w}^{\sigma}$. In what follows, we will need to relate divergence-free vector fields in $D$ to fields satisfying the condition in (3.3b). To this end, we will exploit the following useful lemma.

Lemma 4.1. There exists a constant $C>0$ such that, for every $f \in L^{2}(D)$ with $\int_{D} f(x) d x=0$, there exists a vector field $\mathbf{v} \in H_{0}^{1}(D)$ satisfying

$$
\begin{aligned}
\operatorname{div}_{y} \mathbf{v} & =f \quad \text { and } \\
\|\nabla \mathbf{v}\|_{L^{2}(D)} & \leq C\|f\|_{L^{2}(D)} .
\end{aligned}
$$

Proof. Since $D$ is clearly star-shaped, this is a special case of Lemma III.3.1 on page 116 of [7].

We note that $\mathbf{v}$ is not uniquely determined. In fact, we can add to $\mathbf{v}$ any divergence-free vector field in $D$, satisfying the $H^{1}$ bound above. The vector field $\mathbf{v}$ can be made unique by assuming, for example, that it is curl free. 
Next, we will state and prove several energy-type estimates for $\mathbf{w}^{\sigma}$. These follow from corresponding bounds for $\mathbf{u}^{\sigma}$ thanks to Proposition 3.1 but we derive them here keeping track of the precise dependence on the parameter $\sigma$.

Given a helical vector field $\mathbf{u}_{0} \in H_{0, p e r}^{1}\left(\Omega^{\sigma}\right)$, Proposition 3.1 gives a one-toone correspondence between strong helical solutions of (2.7) and strong solutions of (3.3) with initial data $\mathbf{w}_{0} \in H_{0}^{1}(D)$ satisfying

$$
\operatorname{div}_{y}\left[\left(\mathbf{w}_{0}^{\sigma}\right)_{H}\right]+\frac{2 \pi}{\sigma} E\left[\left(w_{0}^{\sigma, 3}\right)\right]=0,
$$

where $w_{0}^{\sigma, 3}$ refers to the third component of $\mathbf{w}_{0}^{\sigma}$, and $\mathbf{u}_{0}$ and $\mathbf{w}_{0}$ are related via (2.8). In particular, w $\in C\left([0, T), H_{0}^{1}(D)\right)$.

We remark that for any helical vector field $\mathbf{u}_{0}^{\sigma}$ for which the component along the axis of the pipe, $u_{0}^{\sigma, 3}$, is a radial function, the symmetry-reduced constraint on the divergence is in fact simply the divergence-free constraint in $2 \mathrm{D}$ for $\left(\mathbf{w}_{0}^{\sigma}\right)_{H}$, since in this case $E w_{0}^{\sigma, 3} \equiv 0$. In this special case, the analysis is considerably simplified. We may now state our next results, consisting of energy estimates for $\mathbf{w}^{\sigma}$. We split these into two propositions, the first valid for all $\sigma>0$ and the second valid for large $\sigma$.

Proposition 4.2. Given $\sigma>0$, let $\mathrm{w}^{\sigma}$ be a strong solution of (3.3) on the time interval $[0, T)$. Then, for all $t \in(0, T)$, we have that

$$
\begin{array}{r}
\int_{D}\left|\mathbf{w}_{0}^{\sigma}(y)\right|^{2} d y=\int_{D}\left|\mathbf{w}^{\sigma}(t, y)\right|^{2} d y+2 \int_{0}^{t} \int_{D}\left|\nabla \mathbf{w}^{\sigma}(s, y)\right|^{2} d y d s \\
+2 \int_{0}^{t} \int_{D} \frac{4 \pi^{2}}{\sigma^{2}}\left[\left(E\left(w^{\sigma, 3}\right)\right)^{2}+\left|E \mathbf{w}_{H}^{\sigma}-\left(\mathbf{w}_{H}^{\sigma}\right)^{\perp}\right|^{2}\right] d y d s .
\end{array}
$$

Proof. We simply observe that $\mathrm{w}^{\sigma}$ has enough regularity to be a test function in the weak formulation of (3.3), so we are justified in multiplying (3.3) by $\mathbf{w}^{\sigma}$ and integrating over the domain $D$ and, subsequently, in time. This easily yields the desired identity.

Proposition 4.3. Let $1 \leq \sigma<\infty$, and fix $T>0$. Let $\mathbf{u}^{\sigma}$ be a strong helical solutions of (2.7) on the interval $[0, T)$. Let $\mathbf{w}^{\sigma}$ be the corresponding symmetryreduced flow, which solves (3.3). Then the following hold:

(1) There exists $C>0$, independent of $\sigma$, such that

$$
\left\|\partial_{t} \mathbf{w}^{\sigma}\right\|_{L^{2}\left((0, T) ; H^{-1}(D)\right)} \leq C\left(\left\|\mathbf{w}^{\sigma}\right\|_{L^{\infty}\left((0, T) ; L^{2}(D)\right)}+1\right)\left\|\nabla \mathbf{w}^{\sigma}\right\|_{L^{2}\left((0, T) ; L^{2}(D)\right)} .
$$

(2) There exists $C>0$, independent of $\sigma$, such that

$$
\begin{aligned}
& \left\|q^{\sigma}\right\|_{L^{2}\left((0, T) ; L^{2}(D)\right)} \leq C\left(\left\|\partial_{t} \mathbf{w}^{\sigma}\right\|_{L^{2}\left((0, T) ; H^{-1}(D)\right)}\right. \\
& \left.\quad+\left(\left\|\mathbf{w}^{\sigma}\right\|_{L^{\infty}\left((0, T) ; L^{2}(D)\right)}+1\right)\left\|\nabla \mathbf{w}^{\sigma}\right\|_{L^{2}\left((0, T) ; L^{2}(D)\right)}\right) .
\end{aligned}
$$

(3) Moreover, the following scaling holds:

$$
\left\|\mathbf{u}_{0}^{\sigma}\right\|_{L^{2}\left(\Omega^{\sigma}\right)}=\sqrt{\sigma}\left\|\mathbf{w}_{0}^{\sigma}\right\|_{L^{2}(D)},
$$


(4) and we also have

$$
\begin{aligned}
\left\|\nabla_{H} \mathbf{u}_{0}^{\sigma}\right\|_{L^{2}\left(\Omega^{\sigma}\right)} & \leq \sqrt{\sigma}\left\|\nabla \mathbf{w}_{0}^{\sigma}\right\|_{L^{2}(D)}, \\
\left\|\partial_{x_{3}} \mathbf{u}_{0}^{\sigma}\right\|_{L^{2}\left(\Omega^{\sigma}\right)} & \leq \frac{1}{\sqrt{\sigma}}\left\|\mathbf{w}_{0}^{\sigma}\right\|_{H^{1}(D)} .
\end{aligned}
$$

Remark 4.1. As a result of Propositions 4.2 and 4.3 it follows that

$$
\begin{aligned}
& \left\|\mathbf{w}^{\sigma}(t)\right\|_{L^{2}(D)} \leq\left\|\mathbf{w}_{0}^{\sigma}\right\|_{L^{2}(D)}, \quad \text { for each } t \in[0, T] \\
& \left\|\nabla \mathbf{w}^{\sigma}\right\|_{L^{2}\left((0, T) ; L^{2}(D)\right)} \leq C\left\|\mathbf{w}_{0}^{\sigma}\right\|_{L^{2}(D)}, \\
& \left\|\partial_{t} \mathbf{w}^{\sigma}\right\|_{L^{2}\left((0, T) ; H^{-1}(D)\right)} \leq C_{1}\left\|\mathbf{w}_{0}^{\sigma}\right\|_{L^{2}(D)}^{2}+C_{2}\left\|\mathbf{w}_{0}^{\sigma}\right\|_{L^{2}(D)}, \\
& \left\|q^{\sigma}\right\|_{L^{2}\left((0, T) ; L^{2}(D)\right)} \leq C_{1}\left\|\mathbf{w}_{0}^{\sigma}\right\|_{L^{2}(D)}^{2}+C_{2}\left\|\mathbf{w}_{0}^{\sigma}\right\|_{L^{2}(D)},
\end{aligned}
$$

with constants that are uniform in $\sigma$ on $[1,+\infty)$.

Proof. We begin with estimate (1). We recall that $\mathbf{u}^{\sigma}(x)=M^{\sigma}\left(x_{3}\right) \mathbf{w}^{\sigma}\left(t, m^{\sigma}\left(x_{3}\right) x^{\prime}\right)$, where $x^{\prime}=\left(x_{1}, x_{2}\right)$. We exploit the duality between $H^{-1}$ and $H_{0}^{1}$ to compute

$$
\left\|\partial_{t} \mathbf{w}^{\sigma}\right\|_{H^{-1}(D)}=\sup _{\mathbf{\Psi} \neq \mathbf{0}, \boldsymbol{\Psi} \in H_{0}^{1}(D)} \frac{\left\langle\boldsymbol{\Psi}, \mathbf{w}^{\sigma}\right\rangle}{\|\boldsymbol{\Psi}\|_{H_{0}^{1}}} .
$$

To this end, we test the symmetry-reduced equations (3.3) against a (vector) test function $\Psi \in H_{0}^{1}(D)^{3}$ and relate the weak form of the reduced equations to that of the Navier-Stokes equations by constructing an appropriate test function $\boldsymbol{\Phi}$ in $H_{0, p e r}^{1}\left(\Omega^{\sigma}\right)^{3}$ from $\boldsymbol{\Psi}$, as follows:

$$
\boldsymbol{\Phi}(x) \equiv \frac{1}{\sigma} M^{\sigma}\left(x_{3}\right) \mathbf{\Psi}\left(m^{\sigma}\left(x_{3}\right) x^{\prime}\right) .
$$

We recall now as well that $\left(m^{\sigma}\left(x_{3}\right)\right)^{-1} y=x^{\prime}=\left(x_{1}, x_{2}\right)$ by 2.6. We then observe that

$$
\left(M^{\sigma}\left(x_{3}\right)\right)^{T} \mathbf{\Phi}\left(\left(m^{\sigma}\right)^{-1}\left(x_{3}\right) y, x_{3}\right)=\frac{1}{\sigma} \boldsymbol{\Psi}(y),
$$

by the orthogonality of $M^{\sigma}$.

We have that

$$
\begin{gathered}
\int_{D} \boldsymbol{\Psi}(y) \cdot \partial_{t} \mathbf{w}^{\sigma}(t, y) d y \\
=\int_{D} \int_{0}^{\sigma}\left(M^{\sigma}\left(x_{3}\right)\right)^{T} \mathbf{\Phi}\left(\left(m^{\sigma}\right)^{-1}\left(x_{3}\right) y, x_{3}\right) \cdot \partial_{t} \mathbf{w}^{\sigma}(t, y) d x_{3} d y \\
=\int_{\Omega^{\sigma}} \mathbf{\Phi}\left(x_{H}, x_{3}\right) \cdot M^{\sigma}\left(x_{3}\right) \partial_{t} \mathbf{w}^{\sigma}\left(t, m^{\sigma}\left(x_{3}\right) x^{\prime}\right) d x=\int_{\Omega^{\sigma}} \boldsymbol{\Phi}(x) \cdot \partial_{t} \mathbf{u}^{\sigma}(t, x) d x .
\end{gathered}
$$

To bound the $H^{1}$ norm of $\mathbf{u}$, we calculate the derivatives of $\boldsymbol{\Phi}$ to find

$$
\nabla_{H} \boldsymbol{\Phi}(x)=\frac{1}{\sigma} M^{\sigma}\left(x_{3}\right)\left[(D \Psi)\left(m^{\sigma}\left(x_{3}\right) x^{\prime}\right)\right] m^{\sigma}\left(x_{3}\right),
$$




$$
\begin{aligned}
\partial_{x_{3}} \boldsymbol{\Phi}(x)= & \frac{2 \pi}{\sigma^{2}}\left(\partial_{\rho} M^{\sigma}\left(x_{3}\right) \boldsymbol{\Psi}\left(m^{\sigma}\left(x_{3}\right) x^{\prime}\right)+\right. \\
& \left.M^{\sigma}\left(x_{3}\right)\left[(D \boldsymbol{\Psi})\left(m^{\sigma}\left(x_{3}\right) x_{H}\right)\right]\left[\left(\partial_{\rho} m^{\sigma}\right)\left(x_{3}\right) x^{\prime}\right]\right),
\end{aligned}
$$

where $D$ denotes differentiation of a function with respect to its variables and $\rho$ denotes the argument of $M^{\sigma}$ and $m^{\sigma}$. A simple change of variables then gives:

$$
\begin{gathered}
\|\boldsymbol{\Phi}\|_{L^{2}\left(\Omega^{\sigma}\right)}=\frac{1}{\sqrt{\sigma}}\|\boldsymbol{\Psi}\|_{L^{2}(D)} . \\
\left\|\nabla_{H} \boldsymbol{\Phi}\right\|_{L^{2}\left(\Omega^{\sigma}\right)} \leq C \frac{1}{\sqrt{\sigma}}\|\nabla \boldsymbol{\Psi}\|_{L^{2}(D)}, \\
\left\|\partial_{x_{3}} \boldsymbol{\Phi}\right\|_{L^{2}\left(\Omega^{\sigma}\right)} \leq C \frac{1}{\sigma^{3 / 2}}\|\nabla \boldsymbol{\Psi}\|_{L^{2}(D)},
\end{gathered}
$$

with $C$ a constant independent of $\sigma$.

Hence, since $\sigma \geq 1$,

$$
\left\|\partial_{t} \mathbf{w}^{\sigma}(t, \cdot)\right\|_{H^{-1}(D)} \leq C \frac{1}{\sqrt{\sigma}}\left\|\partial_{t} \mathbf{u}^{\sigma}(t, \cdot)\right\|_{H^{-1}\left(\Omega^{\sigma}\right)} .
$$

Next, we estimate the $H^{-1}$ norm of $\partial_{t} \mathbf{u}$ directly from equations (2.7):

$$
\partial_{t} \mathbf{u}^{\sigma}=-\mathbb{P}\left[\left(\mathbf{u}^{\sigma} \cdot \nabla\right) \mathbf{u}^{\sigma}\right]+\mathbb{P}\left[\Delta \mathbf{u}^{\sigma}\right]
$$

where $\mathbb{P}$ denotes the Leray projector onto divergence-free vector fields tangent to $\partial D$ and periodic in $x_{3}$ with period $\sigma$, so that

$$
\begin{gathered}
\left\|\partial_{t} \mathbf{u}^{\sigma}(t, \cdot)\right\|_{H^{-1}\left(\Omega^{\sigma}\right)} \leq C_{1}\left\|\operatorname{div}\left(\mathbf{u}^{\sigma} \otimes \mathbf{u}^{\sigma}\right)(t, \cdot)\right\|_{H^{-1}\left(\Omega^{\sigma}\right)}+C_{2}\left\|\Delta \mathbf{u}^{\sigma}(t, \cdot)\right\|_{H^{-1}\left(\Omega^{\sigma}\right)} \\
\leq C_{1}\left\|\mathbf{u}^{\sigma}(t, \cdot)\right\|_{L^{4}\left(\Omega^{\sigma}\right)}^{2}+C_{2}\left\|\nabla \mathbf{u}^{\sigma}(t, \cdot)\right\|_{L^{2}\left(\Omega^{\sigma}\right)} \\
=C_{1} \sqrt{\sigma}\left\|\mathbf{w}^{\sigma}(t, \cdot)\right\|_{L^{4}(D)}^{2}+C_{2} \frac{1}{\sqrt{\sigma}}\left\|\nabla \mathbf{w}^{\sigma}(t, \cdot)\right\|_{L^{2}(D)},
\end{gathered}
$$

using the helical symmetry expressed by relation (2.8). It follows from (4.4) and the estimates above that

$$
\begin{gathered}
\left\|\partial_{t} \mathbf{w}^{\sigma}(t, \cdot)\right\|_{H^{-1}(D)} \leq C \frac{1}{\sqrt{\sigma}}\left(\sqrt{\sigma}\left\|\mathbf{w}^{\sigma}(t, \cdot)\right\|_{L^{4}(D)}^{2}+\frac{1}{\sqrt{\sigma}}\left\|\nabla \mathbf{w}^{\sigma}(t, \cdot)\right\|_{L^{2}(D)}\right) \\
\leq C\left(\left\|\mathbf{w}^{\sigma}(t, \cdot)\right\|_{L^{2}(D)}\left\|\nabla \mathbf{w}^{\sigma}(t, \cdot)\right\|_{L^{2}(D)}+\left\|\nabla \mathbf{w}^{\sigma}(t, \cdot)\right\|_{L^{2}(D)}\right),
\end{gathered}
$$

where we have used the two-dimensional Ladyzhenskaya inequality (2.1). This concludes the proof of estimate (1).

To prove estimate (2), we deal directly with the equations for $\mathbf{w}^{\sigma}, q^{\sigma}$. Since $p^{\sigma}$, and hence $q^{\sigma}$, is chosen up to a constant, we can assume that

$$
\int_{D} q^{\sigma}(y) d y=0
$$

We again use duality and interpret the $L^{2}$-norm of $q^{\sigma}$ as the dual norm in $\left(L^{2}(D)\right)^{*}$. Consequently, we pick an arbitrary $f \in L^{2}(D)$ such that $\int_{D} f(y) d y=0$ and 
$\|f\|_{L^{2}}=1$. By virtue of Lemma 4.1 there exists $\mathbf{v}_{H} \in H_{0}^{1}(D)$ such that

$$
\begin{aligned}
& \operatorname{div} \mathbf{v}_{H}=f, \\
& \left\|\mathbf{v}_{H}\right\|_{H^{1}(D)} \leq C\|f\|_{L^{2}(D)}=C .
\end{aligned}
$$

We multiply (3.3) by $\mathbf{v}_{H}$ and integrate over $D$ to find:

$$
\begin{gathered}
\int_{D} \mathbf{v}_{H} \cdot\left(\partial_{t} \mathbf{w}_{H}^{\sigma}+\left(\mathbf{w}_{H}^{\sigma} \cdot \nabla_{y}\right) \mathbf{w}_{H}^{\sigma}+\frac{2 \pi}{\sigma} w^{\sigma, 3}\left[E \mathbf{w}_{H}^{\sigma}-\left(\mathbf{w}_{H}^{\sigma}\right)^{\perp}\right]\right) d y \\
=\int_{D} \mathbf{v}_{H} \cdot\left(-\left(\nabla_{y} q^{\sigma}\right)_{H}+\Delta_{y} \mathbf{w}_{H}^{\sigma}+\frac{4 \pi^{2}}{\sigma^{2}}\left[E^{2} \mathbf{w}_{H}^{\sigma}\right.\right. \\
\left.\left.-2 E\left(\mathbf{w}_{H}^{\sigma}\right)^{\perp}-\mathbf{w}_{H}^{\sigma}\right]\right) d y
\end{gathered}
$$

We next perform several integrations by parts, using the divergence constraint for $\mathbf{w}^{\sigma}$ :

$$
\operatorname{div}_{y} \mathbf{w}_{H}+\frac{2 \pi}{\sigma} E w^{3}=0,
$$

together with 4.57, to find

$$
\begin{gathered}
\int_{D} \mathbf{v}_{H} \cdot \partial_{t} \mathbf{w} d y-\int_{D} \mathbf{w}^{\sigma} \cdot\left[\left(\mathbf{w}_{H}^{\sigma} \cdot \nabla_{y}\right) \mathbf{v}_{H}\right] d y \\
-\frac{2 \pi}{\sigma} \int_{D} \mathbf{w}_{H}^{\sigma} \cdot w^{3, \sigma} E \mathbf{v}_{H}+w^{3, \sigma} \mathbf{v}_{H} \cdot\left(\mathbf{w}_{H}^{\sigma}\right)^{\perp} d y \\
=\int_{D} f(y) q^{\sigma}(y) d y-\int_{D} \nabla_{y} \mathbf{v}_{H} \cdot \nabla_{y} \mathbf{w}^{\sigma} d y-\frac{4 \pi^{2}}{\sigma^{2}} \int_{D} E \mathbf{v}_{H} \cdot E \mathbf{w}_{H}^{\sigma} d y \\
+\frac{8 \pi^{2}}{\sigma^{2}} \int_{D} E \mathbf{v}_{H} \cdot\left(\mathbf{w}_{H}^{\sigma}\right)^{\perp} d y-\frac{4 \pi^{2}}{\sigma^{2}} \int_{D} \mathbf{v}_{H} \cdot \mathbf{w}_{H}^{\sigma} d y .
\end{gathered}
$$

By Poincaré's inequality for functions with zero average on $D$, we deduce that

$$
\begin{aligned}
&\left|\int_{D} f q^{\sigma} d y\right| \leq C\left\|\mathbf{v}_{H}\right\|_{H^{1}(D)}\left(\left\|\partial_{t} \mathbf{w}^{\sigma}\right\|_{H^{-1}(D)}+\right. \\
&\left.\left\|\mathbf{w}^{\sigma}\right\|_{L^{4}(D)}^{2}+\left\|\nabla \mathbf{w}^{\sigma}\right\|_{L^{2}(D)}\right),
\end{aligned}
$$

for $C$ a constant independent of $f$ or $\sigma$. Above we exploit that the operator $E=$ $y^{\perp} \cdot \nabla_{y}$ is first order and $\sigma \geq 1$. 
Hence, using that $\left\|\mathbf{v}_{H}\right\|_{H^{1}(D)} \leq C\|f\|_{L^{2}(D)}=C$ from (4.5) and the Ladyzhenskaya inequality again, we find

$$
\left\|q^{\sigma}\right\|_{L^{2}(D)} \equiv\left|\int_{D} f q^{\sigma} d y\right| \leq C\left(\left\|\partial_{t} \mathbf{w}^{\sigma}\right\|_{H^{-1}}+\left\|\mathbf{w}^{\sigma}\right\|_{L^{2}}\left\|\nabla \mathbf{w}^{\sigma}\right\|_{L^{2}}+\left\|\nabla \mathbf{w}^{\sigma}\right\|_{L^{2}}\right)
$$

Finally, squaring both sides of the inequality (4.9) and using Young's inequality, subsequently integrating in time, we arrive at

$$
\begin{aligned}
& \left\|q^{\sigma}\right\|_{L^{2}\left((0, T) ; L^{2}(D)\right)}^{2} \leq C\left(\left\|\partial_{t} \mathbf{w}^{\sigma}\right\|_{L^{2}\left((0, T) ; H^{-1}(D)\right)}^{2}\right. \\
& \left.\quad+\left(\left\|\mathbf{w}^{\sigma}\right\|_{L^{\infty}\left((0, T) ; L^{2}(D)\right)}^{2}+1\right)\left\|\nabla \mathbf{w}^{\sigma}\right\|_{L^{2}\left((0, T) ; L^{2}(D)\right)}^{2}\right) .
\end{aligned}
$$

Identities (3) and (4) follow by a straightforward change of variables, from the relation

which gives by the chain rule,

$$
\mathbf{u}_{0}^{\sigma}(x)=M^{\sigma}\left(x_{3}\right) \mathbf{w}_{0}^{\sigma}\left(m^{\sigma}\left(x_{3}\right) x^{\prime}\right),
$$

$$
\nabla_{H} \mathbf{u}_{0}^{\sigma}=M^{\sigma}\left(x_{3}\right)\left[\left(D \mathbf{w}_{0}^{\sigma}\right)\left(m^{\sigma}\left(x_{3}\right) x^{\prime}\right)\right]\left[m^{\sigma}\left(x_{3}\right)\right],
$$

and

$$
\begin{aligned}
\partial_{x_{3}} \mathbf{u}_{0}^{\sigma}=\frac{2 \pi}{\sigma}\left[\partial_{\rho} M^{\sigma}\left(x_{3}\right) \mathbf{w}_{0}^{\sigma}\left(m^{\sigma}\left(x_{3}\right) x^{\prime}\right)\right. & \\
& \left.+M^{\sigma}\left(x_{3}\right)\left[\left(D \mathbf{w}_{0}^{\sigma}\right)\left(m^{\sigma}\left(x_{3}\right)\right)\right]\left[\partial_{\rho} m^{\sigma}\left(x_{3}\right)\right] x^{\prime}\right] .
\end{aligned}
$$

With these estimates at hand, we are now ready to discuss the limit $\sigma \rightarrow \infty$. We observe that $\sigma$ is not a parameter appearing explicitly in the Navier-Stokes system (2.7). Therefore it is not clear what the limit equations are even at a formal level. The dependence on $\sigma$ is elucidated however in the symmetry-reduced system (3.3), which is equivalent to the original system at the level of strong solutions thanks to Proposition 3.1.

For the reduced system (3.3), formally setting $\sigma=\infty$ produces the following system of equations for a three-component vector function $\mathbf{w}^{\infty}:(0,+\infty) \times D \rightarrow$ $\mathbb{R}^{3}$, with associated pressure $q^{\infty}$ :

$$
\left\{\begin{array}{lc}
\partial_{t} w^{\infty, 1}+\left(w^{\infty, 1} \partial_{y_{1}}+w^{\infty, 2} \partial_{y_{2}}\right) w^{\infty, 1}=-\partial_{y_{1}} q^{\infty}+\left(\partial_{y_{1}}^{2}+\partial_{y_{2}}^{2}\right) w^{\infty, 1}, \\
\partial_{t} w^{\infty, 2}+\left(w^{\infty, 1} \partial_{y_{1}}+w^{\infty, 2} \partial_{y_{2}}\right) w^{\infty, 2}=-\partial_{y_{2}} q^{\infty}+\left(\partial_{y_{1}}^{2}+\partial_{y_{2}}^{2}\right) w^{\infty, 2}, \\
\partial_{t} w^{\infty, 3}+\left(w^{\infty, 1} \partial_{y_{1}}+w^{\infty, 2} \partial_{y_{2}}\right) w^{\infty, 3}=\left(\partial_{y_{1}}^{2}+\partial_{y_{2}}^{2}\right) w^{\infty, 3} \\
\partial_{y_{1}} w^{\infty, 1}+\partial_{y_{2}} w^{\infty, 2}=0, & \text { in }[0,+\infty) \times D ; \\
\mathbf{w}^{\infty}=0, & \text { on }[0,+\infty) \times \partial D ; \\
\mathbf{w}^{\infty}(0, y)=\mathbf{w}_{0}^{\infty}(y), & y \in D .
\end{array}\right.
$$

The initial condition $\mathbf{w}_{0}^{\infty}$ will be taken in $H_{0}^{1}(D)$ and assumed to satisfy:

$$
\partial_{y_{1}} w_{0}^{\infty, 1}+\partial_{y_{2}} w_{0}^{\infty, 2}=0 .
$$

The first two momentum equations are independent of $w^{\infty, 3}$ and together with the fourth equation give precisely the two-dimensional Navier-Stokes equations 
in $D$, where the fluid velocity is identified with $\mathbf{w}_{H}^{\infty}:=\left(w^{\infty, 1}, w^{\infty, 2}, 0\right)$. The third component $w^{\infty, 3}$ is simply advected by the first two and diffused. For this reason, we refer to this flow as a planar flow. Existence and regularity results for the 2D Navier-Stokes equations immediately give existence and uniqueness of the divergence-free vector field $\mathbf{w}_{H}^{\infty} \in C\left([0, T) ; H_{0}^{1}(D)\right) \cap L^{2}\left((0, T) ; H^{2}(D) \cap\right.$ $\left.H_{0}^{1}(D)\right)$ and associated pressure $q^{\infty} \in L^{2}\left((0, T) ; H^{1}(D)\right) \cap C^{\infty}((0, T) \times D)$ for any initial condition $\mathbf{w}_{H}^{\infty}(0) \in H_{0}^{1}(D)$ satisfying (4.12), and any $T>0$. In fact, $\mathbf{w}_{H}^{\infty}$ is smooth for $t>0$. Consequently, the advection-diffusion equation for $w^{\infty, 3}$ admits a unique solution, which belongs to the same class (see e.g. Proposition 2.7 in [13] and Theorem 3.10 in [16].) We refer to the three-component vector function

$$
\mathbf{w}^{\infty} \in C\left([0, T) ; H_{0}^{1}(D)\right) \cap L^{2}\left((0, T) ; H^{2}(D) \cap H_{0}^{1}(D)\right) \cap C^{\infty}((0, T) \times D),
$$

as the unique strong solution of problem 4.11.

The System (4.11) gives the so-called two-dimensional, three-component NavierStokes equations (also known as the $2 \frac{1}{2} D$ Navier-Stokes equations, see [13].) We can uniquely associate to $\mathbf{w}^{\infty}$ a solution $\mathbf{u}^{\infty}$ of the Navier-Stokes equations in $\Omega$ with initial data $\mathbf{u}_{0}^{\infty}$ by:

$$
\begin{array}{lr}
\mathbf{u}^{\infty}(t, x):=\mathbf{w}^{\infty}\left(t, x^{\prime}\right), & x^{\prime} \in D, t>0, \\
\mathbf{u}_{0}^{\infty}(x):=\mathbf{w}_{0}^{\infty}\left(x^{\prime}\right), & x^{\prime} \in D \\
p^{\infty}(t, x):=q^{\infty}\left(t, x^{\prime}, 0\right), & x^{\prime} \in D, t>0,
\end{array}
$$

with $x^{\prime}=\left(x_{1}, x_{2}\right)$. It is immediate to see that $\mathbf{u}^{\infty}$ and $p^{\infty}$ have at least the same regularity as $\mathbf{w}^{\infty}$ and $q^{\infty}$. We will refer to $\mathbf{u}^{\infty}$ as the $2 \frac{1}{2} \mathrm{D}$ solution of the NavierStokes system (2.7) in $\Omega$ with associated pressure $p^{\infty}$.

To obtain a relationship with the original problem (2.7), at least at a formal level, we observe that, if we take the limit $\sigma \rightarrow \infty$ in (2.5), thanks to (2.2) and (2.6), we have the identification:

$$
\mathbf{u}^{\infty}(t, x)=\mathbf{w}^{\infty}\left(t, x^{\prime}\right) \equiv \lim _{\sigma \rightarrow \infty} \mathbf{u}^{\sigma}(t, x)
$$

Above, we have naturally identified the cross section of the cylinder $\Omega$ at height $x_{3}=0$ with $D$ and $x^{\prime}=\left(x_{1}, x_{2}\right)$ with $y$. We will use the identities and estimates established in Proposition 4.3 valid for all $1 \leq \sigma<\infty$, to establish an estimate for the difference between $\mathbf{w}^{\sigma}$ and $\mathbf{w}^{\infty}$. One difficulty in studying the limit $\sigma \rightarrow \infty$ is that $\mathbf{w}_{H}^{\infty}$ is divergence free, while $\mathbf{w}_{H}^{\sigma}$ satisfies a divergence constraint that is $\sigma$ dependent.

Proposition 4.4. Let $\mathbf{w}_{0}^{\infty} \in H_{0}^{1}(D)$ satisfy (4.12). Given $\sigma \geq 1$, let $\mathbf{w}_{0}^{\sigma} \in H_{0}^{1}(D)$ satisfy 4.1). Let $\mathbf{w}^{\infty}$ be the unique strong solution of 4.11) with initial data $\mathbf{w}_{0}^{\infty}$, and let $\mathbf{w}^{\sigma}$ be the unique regular solution of (3.3) with initial data $\mathbf{w}_{0}^{\sigma}$ on the time interval $(0, T), T>0$. Set:

$$
\Theta^{\sigma} \equiv \mathbf{w}^{\sigma}-\mathbf{w}^{\infty}
$$


Then, for all $0<t<T$,

$$
\begin{aligned}
\int_{D}\left|\Theta^{\sigma}(t, y)\right|^{2} d y & +\int_{0}^{t} \int_{D}\left|\nabla \Theta^{\sigma}(s, y)\right|^{2} d s d y \\
& \leq C\left(t,\left\|\mathbf{w}_{0}^{\infty}\right\|_{L^{2}}^{2},\left\|\mathbf{w}_{0}^{\sigma}\right\|_{L^{2}}^{2}\right)\left(\left\|\Theta_{0}^{\sigma}\right\|_{L^{2}}^{2}+\frac{1}{\sigma}\right) .
\end{aligned}
$$

Proof. Since $\mathrm{w}^{\sigma}$ is a strong solution of (3.3) and $\mathrm{w}^{\infty}$ is of (4.11) on the interval $[0, T)$, there exist functions $q^{\sigma}$ and $q^{\infty} \in L^{1}\left((0, T) ; H^{1}(D)\right)$ enforcing the divergence constraints. If we set $r^{\sigma}=q^{\infty}-q^{\sigma}$, then $\Theta^{\sigma}$ satisfies the following set of equations on $(0, T) \times D$ :

$$
\left\{\begin{array}{l}
\partial_{t} \boldsymbol{\Theta}^{\sigma}+\left(\mathbf{w}_{H}^{\sigma} \cdot \nabla_{y}\right) \boldsymbol{\Theta}^{\sigma}+\left(\Theta_{H}^{\sigma} \cdot \nabla_{y}\right) \mathbf{w}^{\infty}+\frac{2 \pi}{\sigma} w^{\sigma, 3}\left[E \mathbf{w}^{\sigma}-\left(\mathbf{w}_{H}^{\sigma}\right)^{\perp}\right] \\
=-\left(\nabla_{y} r^{\sigma}\right)_{H}-\frac{2 \pi}{\sigma} E q^{\sigma} \mathbf{e}_{3}+\Delta_{y} \boldsymbol{\Theta}^{\sigma}+\frac{4 \pi^{2}}{\sigma^{2}}\left[E^{2} \mathbf{w}^{\sigma}-2 E\left(\mathbf{w}_{H}^{\sigma}\right)^{\perp}-\mathbf{w}_{H}^{\sigma}\right] \\
\operatorname{div}_{y} \boldsymbol{\Theta}_{H}^{\sigma}+\frac{2 \pi}{\sigma} E w^{\sigma, 3}=0
\end{array}\right.
$$

where $E$ is again the differential operator $y^{\perp} \cdot \nabla_{y}$ defined in (2.4). These equations are complemented by the initial condition

$$
\boldsymbol{\Theta}_{0}^{\sigma}:=\boldsymbol{\Theta}^{\sigma}(0)=\mathbf{w}_{0}^{\sigma}-\mathbf{w}_{0}^{\infty} \in H_{0}^{1}(D)
$$

and no-slip boundary conditions on $\partial D$.

We observe that $\Theta^{\sigma}$ has enough regularity to be a test function for the weak formulation of (4.17). In particular, $\partial_{t} \Theta^{\sigma} \in L^{2}\left((0, T), L^{2}(D)\right)$. The weak form, after rearranging the terms and integrating by parts, gives:

$$
\begin{aligned}
& \frac{1}{2} \frac{d}{d t} \int_{D}\left|\boldsymbol{\Theta}^{\sigma}\right|^{2} d y+\int_{D}\left|\nabla \boldsymbol{\Theta}^{\sigma}\right|^{2} d y=-\int_{D} \boldsymbol{\Theta}^{\sigma} \cdot\left[\left(\mathbf{w}_{H}^{\sigma} \cdot \nabla_{y}\right) \boldsymbol{\Theta}^{\sigma}\right] d y \\
& -\int_{D} \boldsymbol{\Theta}^{\sigma} \cdot\left[\left(\boldsymbol{\Theta}_{H}^{\sigma} \cdot \nabla_{y}\right) \mathbf{w}^{\infty}\right] d y-\frac{2 \pi}{\sigma} \int_{D} \boldsymbol{\Theta}^{\sigma} \cdot\left[w^{\sigma, 3}\left(E \mathbf{w}^{\sigma}-\left(\mathbf{w}_{H}^{\sigma}\right)^{\perp}\right)\right] d y \\
& +\frac{4 \pi^{2}}{\sigma^{2}} \int_{D} \boldsymbol{\Theta}^{\sigma} \cdot\left[E^{2} \mathbf{w}^{\sigma}-2 E\left(\mathbf{w}_{H}^{\sigma}\right)^{\perp}-\mathbf{w}_{H}^{\sigma}\right] d y \\
& -\int_{D} \boldsymbol{\Theta}^{\sigma} \cdot\left[\left(\nabla_{y} r^{\sigma}\right)_{H}+\frac{2 \pi}{\sigma} E q^{\sigma} \mathbf{e}_{3}\right] d y \equiv-I_{1}-I_{2}-I_{3}+I_{4}-I_{5}
\end{aligned}
$$

We estimate each of the five integrals on the right-hand side. Since $\mathbf{w}^{\sigma}$ is a strong solution and in view of estimates 4.3 for $\mathbf{w}^{\sigma}$, all norms appearing in the bounds to follow are finite and all constants $C$ are uniform in $\sigma \in[1,+\infty)$. We 
have:

$$
\begin{aligned}
& \left|I_{1}\right| \leq \frac{2 \pi}{\sigma} \int_{D} \frac{1}{2}\left|\Theta^{\sigma}\right|^{2}\left|\nabla \mathbf{w}^{\sigma}\right| d y \\
& \left|I_{2}\right| \leq \int_{D}\left|\Theta^{\sigma}\right|^{2}\left|\nabla \mathbf{w}^{\infty}\right| d y \\
& \left|I_{3}\right| \leq \frac{2 \pi}{\sigma} \int_{D}\left|\Theta^{\sigma}\right|\left|\mathbf{w}^{\sigma}\right|\left(\left|\nabla \mathbf{w}^{\sigma}\right|+\left|\mathbf{w}^{\sigma}\right|\right) d y \\
& \left|I_{4}\right| \leq \frac{4 \pi^{2}}{\sigma^{2}}\left[\int_{D}\left|\nabla \Theta^{\sigma}\right|\left|\nabla \mathbf{w}^{\sigma}\right| d y+\int_{D}\left|\Theta^{\sigma}\right|\left(\left|\nabla \mathbf{w}^{\sigma}\right|+\left|\mathbf{w}^{\sigma}\right|\right) d y\right], \\
& \left|I_{5}\right|=\left|\frac{2 \pi}{\sigma} \int_{D}\left[q^{\infty} E\left(w^{\sigma, 3}\right)-q^{\sigma} E\left(w^{\infty, 3}\right)\right] d y\right| \leq \frac{2 \pi}{\sigma} \int_{D}\left(\left|\nabla \mathbf{w}^{\sigma}\right|\left|q^{\infty}\right|+\left|\nabla \mathbf{w}^{\infty}\right|\left|q^{\sigma}\right|\right) d y .
\end{aligned}
$$

We bound further each integral $I_{i}, i=1, \ldots, 5$, using repeatedly the Ladyzhenskaya inequality 2.1], Cauchy-Schwartz and Young's inequalities:

$$
\begin{aligned}
& \left|I_{1}\right| \leq \frac{C}{\sigma}\left\|\Theta^{\sigma}\right\|_{L^{4}(D)}^{2}\left\|\nabla \mathbf{w}^{\sigma}\right\|_{L^{2}(D)} \leq \frac{C}{\sigma}\left(\left\|\Theta^{\sigma}\right\|_{L^{2}(D)}^{2}\left\|\nabla \mathbf{w}^{\sigma}\right\|_{L^{2}(D)}^{2}+\left\|\nabla \Theta^{\sigma}\right\|_{L^{2}(D)}^{2}\right), \\
& \left|I_{2}\right| \leq\left\|\Theta^{\sigma}\right\|_{L^{4}(D)}^{2}\left\|\nabla \mathbf{w}^{\infty}\right\|_{L^{2}(D)} \leq \frac{1}{2}\left\|\Theta^{\sigma}\right\|_{L^{2}(D)}^{2}\left\|\nabla \mathbf{w}^{\infty}\right\|_{L^{2}(D)}^{2}+\frac{1}{2}\left\|\nabla \boldsymbol{\Theta}^{\sigma}\right\|_{L^{2}(D)}^{2}, \\
& \left|I_{3}\right| \leq \frac{C}{\sigma}\left(\left\|\Theta^{\sigma}\right\|_{L^{2}(D)}\left\|\mathbf{w}^{\sigma}\right\|_{L^{4}(D)}^{2}+\left\|\Theta^{\sigma}\right\|_{L^{4}(D)}\left\|\nabla \mathbf{w}^{\sigma}\right\|_{L^{2}(D)}\left\|\mathbf{w}^{\sigma}\right\|_{L^{4}(D)}\right) \\
& \leq \frac{C}{\sigma}\left(\left\|\Theta^{\sigma}\right\|_{L^{2}(D)}^{2}\left\|\mathbf{w}^{\sigma}\right\|_{L^{2}(D)}^{2}+\left\|\nabla \mathbf{w}^{\sigma}\right\|_{L^{2}(D)}^{2}+\left\|\Theta^{\sigma}\right\|_{L^{2}(D)}^{1 / 2}\left\|\nabla \Theta^{\sigma}\right\|_{L^{2}(D)}^{1 / 2}\left\|\nabla \mathbf{w}^{\sigma}\right\|_{L^{2}(D)}^{3 / 2}\left\|\mathbf{w}^{\sigma}\right\|_{L^{2}(D)}^{1 / 2}\right) \\
& \leq \frac{C}{\sigma}\left(\left\|\Theta^{\sigma}\right\|_{L^{2}(D)}^{2}\left\|\mathbf{w}^{\sigma}\right\|_{L^{2}(D)}^{2}+\left\|\nabla \mathbf{w}^{\sigma}\right\|_{L^{2}(D)}^{2}+\left\|\Theta^{\sigma}\right\|_{L^{2}(D)}^{2}\left\|\nabla \Theta^{\sigma}\right\|_{L^{2}(D)}^{2}\left\|\mathbf{w}^{\sigma}\right\|_{L^{2}(D)}^{2}+\left\|\nabla \mathbf{w}^{\sigma}\right\|_{L^{2}(D)}^{2}\right) \\
& \leq \frac{C}{\sigma}\left[\left\|\Theta^{\sigma}\right\|_{L^{2}(D)}^{2}\left\|\mathbf{w}^{\sigma}\right\|_{L^{2}(D)}^{2}+\left\|\nabla \mathbf{w}^{\sigma}\right\|_{L^{2}(D)}^{2}\right. \\
& \left.+\left(\left\|\mathbf{w}^{\sigma}\right\|_{L^{2}(D)}^{2}+\|\mathbf{u}\|_{L^{2}(D)}^{2}\right)\left\|\nabla \Theta^{\sigma}\right\|_{L^{2}(D)}^{2}\left\|\mathbf{w}^{\sigma}\right\|_{L^{2}(D)}^{2}+\left\|\nabla \mathbf{w}^{\sigma}\right\|_{L^{2}(D)}^{2}\right], \\
& \left|I_{4}\right| \leq \frac{C}{\sigma^{2}}\left(\left\|\nabla \Theta^{\sigma}\right\|_{L^{2}}\left\|\nabla \mathbf{w}^{\sigma}\right\|_{L^{2}(D)}+\left\|\Theta^{\sigma}\right\|_{L^{2}(D)}\left\|\nabla \mathbf{w}^{\sigma}\right\|_{L^{2}(D)}+\left\|\Theta^{\sigma}\right\|_{L^{2}(D)}\left\|\mathbf{w}^{\sigma}\right\|_{L^{2}(D)}\right) \\
& \leq \frac{C}{\sigma^{2}}\left(\left\|\nabla \boldsymbol{\Theta}^{\sigma}\right\|_{L^{2}(D)}^{2}+\left\|\nabla \mathbf{w}^{\sigma}\right\|_{L^{2}(D)}^{2}+\left\|\Theta^{\sigma}\right\|_{L^{2}(D)}^{2}+\left\|\mathbf{w}^{\sigma}\right\|_{L^{2}(D)}^{2}\right), \\
& \left|I_{5}\right| \leq \frac{C}{\sigma}\left(\left\|q^{\infty}\right\|_{L^{2}(D)}\left\|\nabla \mathbf{w}^{\sigma}\right\|_{L^{2}(D)}+\left\|q^{\sigma}\right\|_{L^{2}(D)}\left\|\nabla \mathbf{w}^{\infty}\right\|_{L^{2}}(D)\right) \\
& \leq \frac{C}{\sigma}\left(\left\|q^{\infty}\right\|_{L^{2}(D)}^{2}+\left\|\nabla \mathbf{w}^{\sigma}\right\|_{L^{2}(D)}^{2}+\left\|q^{\sigma}\right\|_{L^{2}(D)}^{2}+\left\|\nabla \mathbf{w}^{\infty}\right\|_{L^{2}(D)}^{2}\right) .
\end{aligned}
$$

Inserting estimates (4.19) - 4.23) into identity (4.18) yields:

$$
\frac{d}{d t}\left\|\Theta^{\sigma}\right\|_{L^{2}(D)}^{2}+\left\|\nabla \boldsymbol{\Theta}^{\sigma}\right\|_{L^{2}(D)}^{2} \leq\left\|\nabla \boldsymbol{\Theta}^{\sigma}\right\|_{L^{2}(D)}^{2} .
$$




$$
\begin{gathered}
\left(\frac{C}{\sigma}+\frac{1}{2}+\frac{C}{\sigma}\left\|\mathbf{w}^{\sigma}\right\|_{L^{2}(D)}^{4}+\frac{C}{\sigma}\left\|\mathbf{w}^{\sigma}\right\|_{L^{2}(D)}^{2}\left\|\mathbf{w}^{\infty}\right\|_{L^{2}(D)}^{2}+\frac{C}{\sigma^{2}}\right)+\left\|\Theta^{\sigma}\right\|_{L^{2}(D)}^{2} \\
\left(\frac{C}{\sigma}\left\|\nabla \mathbf{w}^{\sigma}\right\|_{L^{2}(D)}^{2}+\frac{C}{\sigma}\left\|\nabla \mathbf{w}^{\infty}\right\|_{L^{2}(D)}^{2}+\frac{C}{\sigma}\left\|\mathbf{w}^{\sigma}\right\|_{L^{2}(D)}^{2}+\frac{1}{2}\left\|\nabla \mathbf{w}^{\infty}\right\|_{L^{2}(D)}^{2}+\frac{C}{\sigma^{2}}\right) \\
+\left(\frac{C}{\sigma}\left\|\nabla \mathbf{w}^{\sigma}\right\|_{L^{2}(D)}^{2}+\frac{C}{\sigma^{2}}\left\|\nabla \mathbf{w}^{\sigma}\right\|_{L^{2}(D)}^{2}+\frac{C}{\sigma^{2}}\left\|\mathbf{w}^{\sigma}\right\|_{L^{2}(D)}^{2}+\frac{C}{\sigma}\left\|\nabla \mathbf{w}^{\infty}\right\|_{L^{2}(D)}^{2}\right. \\
\left.+\frac{C}{\sigma}\left\|q^{\infty}\right\|_{L^{2}(D)}^{2}+\frac{C}{\sigma}\left\|q^{\sigma}\right\|_{L^{2}(D)}^{2}\right) .
\end{gathered}
$$

Thanks again to the regularity of $\mathbf{w}^{\infty}$, i.e.,

$$
\mathbf{w}^{\infty} \in C\left([0, T) ; H_{0}^{1}(D)\right) \cap L^{2}\left((0, T) ; H^{2}(D) \cap H_{0}^{1}(D)\right),
$$

and estimates (4.3) for $\mathbf{w}^{\sigma}$, we can now choose $\sigma$ large enough such that

$$
\frac{C}{\sigma}+\frac{1}{2}+\frac{C}{\sigma}\left\|\mathbf{w}^{\sigma}\right\|_{L^{2}(D)}^{4}+\frac{C}{\sigma}\left\|\mathbf{w}^{\sigma}\right\|_{L^{2}(D)}^{2}\left\|\mathbf{w}^{\infty}\right\|_{L^{2}(D)}^{2}+\frac{C^{2}}{\sigma}<\frac{3}{4} .
$$

We will rewrite (4.24) as a differential inequality in order to apply Grönwall's Lemma. To this end, we introduce the functions

$$
f(t)=\frac{C}{\sigma}\left\|\nabla \mathbf{w}^{\sigma}\right\|_{L^{2}}^{2}+\left(\frac{C}{\sigma}+\frac{1}{2}\right)\left\|\nabla \mathbf{w}^{\sigma}\right\|_{L^{2}}^{2}+\frac{C}{\sigma}\left\|\mathbf{w}^{\sigma}\right\|_{L^{2}}^{2}+\frac{C}{\sigma^{2}}
$$

and

$$
\begin{aligned}
g(t)=\frac{C}{\sigma}\left\|\nabla \mathbf{w}^{\sigma}(t)\right\|_{L^{2}}^{2}+\frac{C}{\sigma^{2}}\left\|\nabla \mathbf{w}^{\sigma}(t)\right\|_{L^{2}}^{2}+\frac{C}{\sigma^{2}}\left\|\mathbf{w}^{\sigma}(t)\right\|_{L^{2}}^{2} \\
+\frac{C}{\sigma}\left\|\nabla \mathbf{w}^{\infty}(t)\right\|_{L^{2}}^{2}+\frac{C}{\sigma}\left\|q^{\infty}(t)\right\|_{L^{2}}^{2}+\frac{C}{\sigma}\left\|q^{\sigma}(t)\right\|_{L^{2}}^{2} .
\end{aligned}
$$

We also set

$$
z(t)=\left\|\Theta^{\sigma}(t)\right\|_{L^{2}}^{2} .
$$

With this notation the differential inequality above becomes

$$
\frac{d}{d t} z \leq f(t) z+g(t)
$$

so that, by Grönwall's Lemma we conclude that

$$
z(t) \leq \exp \left\{\int_{0}^{t} f(s) d s\right\} z(0)+\int_{0}^{t} \exp \left\{\int_{s}^{t} f(\tau) d \tau\right\} g(s) d s .
$$

Next, standard energy estimates for the 2D Navier-Stokes equations along with similar energy estimates for advection-diffusion equations, using that $\mathbf{w}_{H}^{\infty}$ is divergencefree, give:

$$
\int_{0}^{t}\left\|\nabla \mathbf{w}^{\infty}(s)\right\|_{L^{2}(D)}^{2} d s \leq\left\|\mathbf{w}_{0}^{\infty}\right\|_{L^{2}(D)}^{2} .
$$

We employ once again the estimates (4.3) in Remark 4.1 to deduce that

$$
\int_{0}^{t} f(s) d s \leq C\left(\left\|\mathbf{w}_{0}^{\infty}\right\|_{L^{2}}^{2}+\frac{1}{\sigma}\left\|\mathbf{w}_{0}^{\sigma}\right\|_{L^{2}}^{2}\right)
$$




$$
\int_{0}^{t} g(s) d s \leq \frac{C}{\sigma}\left(\left\|\mathbf{w}_{0}^{\infty}\right\|_{L^{2}}^{2}+\left\|\mathbf{w}_{0}^{\sigma}\right\|_{L^{2}}^{2}\right)
$$

Hence we arrive at the estimate, using that $\sigma \geq 1$,

$$
\left\|\boldsymbol{\Theta}^{\sigma}\right\|_{L^{2}}^{2} \leq C\left(\left\|\mathbf{w}_{0}^{\infty}\right\|_{L^{2}}^{2},\left\|\mathbf{w}_{0}^{\sigma}\right\|_{L^{2}}^{2}\right)\left\|\boldsymbol{\Theta}_{0}^{\sigma}\right\|_{L^{2}}^{2}+\frac{C}{\sigma}\left(\left\|\mathbf{w}_{0}^{\infty}\right\|_{L^{2}}^{2}+\left\|\mathbf{w}_{0}^{\sigma}\right\|_{L^{2}}^{2}\right) .
$$

This estimate, together with the choice of $\sigma$, produces, upon integrating the differential inequality (4.24) in time, the desired result.

Before formulating our main results concerning the limit $\sigma \rightarrow \infty$, we note a consequence of Proposition 4.4 namely, there may be more than one $2 \frac{1}{2} \mathrm{D}$ flow within a certain distance to a given helical flow $\mathbf{w}^{\sigma}$. This non-uniqueness will be apparent later, since a correction to the initial data $\mathbf{w}_{0}^{\sigma}$ will be needed to enforce the divergence-free condition for $\mathbf{w}_{0}^{\infty}$.

We start with a simpler result, describing a way in which solutions of the twodimensional, three-component Navier-Stokes equations can be approximated by suitable helical solutions of the three-dimensional Navier-Stokes equations. More precisely, suppose we are initially given a vector function

$$
\mathbf{w}_{0}^{\infty}=\left(w_{0}^{\infty, 1}, w_{0}^{\infty, 2}, w_{0}^{\infty, 3}\right) \in H_{0}^{1}(D)
$$

that satisfies the divergence-free constraint 4.12). Let $\mathrm{w}^{\infty}$ be the unique strong solution of 4.11 with pressure $q^{\infty}$. Recall that we can uniquely associate to $\mathrm{w}^{\infty}$ a solution $\mathbf{u}^{\infty}$ of the Navier-Stokes equations in $\Omega$ with initial data $\mathbf{u}_{0}^{\infty}$ via (4.13). We will construct a $\sigma$-dependent correction to $\mathbf{w}_{0}^{\infty}, \mathbf{v}_{0}^{\sigma}$, using Lemma 4.1, so that the resulting field $\mathrm{w}_{0}^{\sigma}$, given in (4.28) below, satisfies 4.1) and, hence, can be taken as initial data for the reduced helical equations (3.3).

We first observe that, since $\left.\mathbf{w}_{0}^{\infty}\right|_{\partial D} \equiv 0$ and $\operatorname{div}_{y} y^{\perp} \equiv 0$,

$$
\int_{D} y^{\perp} \cdot \nabla_{y} w_{0}^{\infty, 3} d y=\int_{D} \operatorname{div}_{y}\left(y^{\perp} w_{0}^{\infty, 3}\right) d y=0 .
$$

Therefore, by Lemma 4.1 there exists a solution $\mathbf{v}_{0}^{\sigma}=\left(v_{0}^{\sigma, 1}, v_{0}^{\sigma, 2}\right) \in H_{0}^{1}(D)$ to the problem

$$
\operatorname{div}_{y} \mathbf{v}_{0}^{\sigma}=-\frac{2 \pi}{\sigma} E w_{0}^{\infty, 3}
$$

such that

$$
\left\|\mathbf{v}_{0}^{\sigma}\right\|_{H^{1}} \leq C \frac{1}{\sigma}\left\|E w_{0}^{\infty, 3}\right\|_{L^{2}(D)} \leq \frac{C}{\sigma}\left\|\mathbf{w}_{0}^{\infty}\right\|_{H^{1}(D)},
$$

where we recall that $E=y^{\perp} \cdot \nabla_{y}$.

Next, we introduce the three-component vector function

$$
\mathbf{w}_{0}^{\sigma}=\mathbf{w}_{0}^{\infty}+\left(\mathbf{v}_{0}^{\sigma}, 0\right) \in H_{0}^{1}(D),
$$

which by construction satisfies (4.1), since $w^{\sigma, 3}=w^{\infty, 3}$. We will take $\mathbf{w}_{0}^{\sigma}$ so constructed as initial data for (3.3). We are now ready to state our first theorem.

Theorem 4.5. Fix $\sigma \geq 1$. Let $\mathbf{w}_{0}^{\infty} \in H_{0}^{1}(D)$ satisfy (4.12). Let $\mathbf{w}^{\infty}$ be the unique strong solution of 4.11) with initial data $\mathbf{w}_{0}^{\infty}$. Let $\mathbf{u}^{\infty}$ be the unique $2 \frac{1}{2} D$ solution of the Navier-Stokes equations (2.7) associated to $\mathbf{w}^{\infty}$ via (4.13). Let $\mathbf{w}_{0}^{\sigma}$ be given 
by (4.28) for a choice of $\mathbf{v}_{0}^{\sigma}$ solution of (4.26), and denote by $\mathbf{w}^{\sigma}$ the strong solution of (3.3) with initial data $\mathbf{w}_{0}^{\sigma}$. Let $\mathbf{u}_{0}^{\sigma}$ be the associated strong helical solution of the Navier-Stokes equations (2.7) given by Proposition 3.1] Then, for any fixed $T>0$,

$$
\begin{aligned}
& \left\|\mathbf{u}^{\sigma}\left(t, \cdot, x_{3}=0\right)-\mathbf{u}^{\infty}\left(t, \cdot, x_{3}=0\right)\right\|_{L^{2}(D)} \leq \frac{C}{\sqrt{\sigma}}, \quad \text { for all } 0<t<T, \\
& \left\|\left.\nabla_{H} \mathbf{u}^{\sigma}\right|_{x_{3}=0}-\left.\nabla_{H} \mathbf{u}^{\infty}\right|_{x_{3}=0}\right\|_{L^{2}\left(0, T ; L^{2}(D)\right)} \leq \frac{C}{\sqrt{\sigma}},
\end{aligned}
$$

where $C$ is independent of $\sigma \in[1, \infty)$.

Proof. Since by hypothesis, both $\mathbf{u}^{\sigma}$ and $\mathbf{u}^{\infty} \in C\left([0, T) ; H_{0, p e r}^{1}\left(\Omega^{\sigma}\right)\right.$

$\cap L^{2}\left((0, T) ; H_{p e r}^{2}\left(\Omega^{\sigma}\right) \cap H_{0, p e r}^{1}\left(\Omega^{\sigma}\right)\right)$, the traces $\left.\mathbf{u}^{\sigma}\right|_{x_{3}=0}(t)$ and $\left.\mathbf{u}^{\infty}\right|_{x_{3}=0}(t)$ are well defined as elements of $L^{2}(D)$ for all $0 \leq t<T$, while the traces $\left.\nabla \mathbf{u}^{\sigma}\right|_{x_{3}=0}$ and $\left.\nabla \mathbf{u}^{\infty}\right|_{x_{3}=0}$ are well defined as elements of $L^{2}\left((0, T) ; L^{2}(D)\right)$.

We continue by showing that

$$
\begin{aligned}
\left\|\mathbf{w}_{0}^{\sigma}-\mathbf{w}_{0}^{\infty}\right\|_{L^{2}(D)} & \leq \frac{C}{\sqrt{\sigma}} \quad \text { and } \\
\left\|\mathbf{w}_{0}^{\sigma}\right\|_{L^{2}(D)} & \leq C,
\end{aligned}
$$

with constants $C$ uniform in $\sigma \in[1, \infty)$. To see that the first statement (4.30) holds true, we note that

$$
\mathbf{w}_{0}^{\sigma}-\mathbf{w}_{0}^{\infty}=\left(\mathbf{v}_{0}^{\sigma}, 0\right),
$$

where $\mathbf{v}_{0}^{\sigma}$ is a solution of (4.26) and satisfies (4.27).

Hence,

$$
\left\|\mathbf{w}_{0}^{\sigma}-\mathbf{w}_{0}^{\infty}\right\|_{L^{2}(D)}=\left\|\mathbf{v}_{0}^{\sigma}\right\|_{L^{2}(D)} \leq\left\|\mathbf{v}_{0}^{\sigma}\right\|_{H^{1}(D)} \leq \frac{C}{\sigma}\left\|\mathbf{w}_{0}^{\infty}\right\|_{H^{1}(D)} .
$$

The second statement (4.31) follows immediately from the first.

Then, Proposition 4.4 gives that

$$
\left\|\mathbf{w}^{\sigma}(t, \cdot)-\mathbf{w}^{\infty}(t, \cdot)\right\|_{L^{2}(D)} \leq \frac{C}{\sqrt{\sigma}}, \quad \text { for almost all } 0<t<T
$$

and

$$
\left\|\nabla_{H} \mathbf{w}^{\sigma}-\nabla_{H} \mathbf{w}^{\infty}\right\|_{L^{2}\left(0, T ; L^{2}(D)\right)} \leq \frac{C}{\sqrt{\sigma}},
$$

again with constants $C$ that do not depend on $\sigma \geq 1$.

Next, the proof of Proposition 3.1 shows that the helical solution $\mathbf{u}^{\sigma}$ of 2.7) with initial condition $\mathbf{u}_{0}^{\sigma}$ related to $\mathbf{w}_{0}^{\sigma}$ via (2.8) is given by

$$
\mathbf{u}^{\sigma}\left(t, x^{\prime}, x_{3}\right)=M^{\sigma}\left(x_{3}\right) \mathbf{w}^{\sigma}\left(t, m^{\sigma}\left(x_{3}\right) x^{\prime}\right),
$$

for $t>0$, where $x^{\prime}=\left(x_{1}, x_{2}\right)$, so that in particular:

$$
\mathbf{u}^{\sigma}\left(t, x_{H}\right)=\mathbf{w}^{\sigma}\left(t, x^{\prime}\right), \quad \nabla_{H} \mathbf{u}^{\sigma}\left(t, x_{H}\right)=\nabla_{x^{\prime}} \mathbf{w}^{\sigma}\left(t, x^{\prime}\right) .
$$

From 4.13, it also immediately follows that

$$
\mathbf{u}^{\infty}\left(t, x_{H}\right)=\mathbf{w}^{\infty}\left(t, x^{\prime}\right), \quad \nabla_{x} \mathbf{u}^{\infty}\left(t, x_{H}\right)=\nabla_{H} \mathbf{u}^{\infty}\left(t, x_{H}\right)=\nabla_{x^{\prime}} \mathbf{w}^{\sigma}\left(t, x^{\prime}\right) .
$$

Then, estimate (4.29) is a straightforward consequence of (4.32) and 4.33). 
Remark 4.2. It is natural to derive bounds of traces at $x_{3}=0$ in view of (4.14). In fact, recalling that $\mathbf{u}^{\sigma}$ is smooth in $x \in \Omega^{\sigma}$ for $t>0$, a simple argument, using a Taylor's expansion for $\mathbf{u}^{\sigma}$ in $0 \leq x^{3} / \sigma<1$, centered at 0 with $x^{\prime} \in D$ fixed, shows that for a given fixed $t$,

$$
\left|\mathbf{u}^{\sigma}(t, x)-\mathbf{u}^{\infty}(t, x)\right|=\left|\mathbf{w}^{\sigma}\left(t, x^{\prime}\right)-\mathbf{w}^{\infty}\left(t, x^{\prime}\right)\right|+O\left(\frac{\left|x_{3}\right|}{\sigma}\right)
$$

with bounds that depend on $\left|\mathbf{w}^{\sigma}\left(x^{\prime}\right)\right|$ and $\left|\nabla_{y} \mathbf{w}^{\sigma}\left(x^{\prime}\right)\right|$. Therefore, an argument similar to that of the proof of Theorem 4.5 above gives:

$$
\begin{aligned}
& \left\|\mathbf{u}^{\sigma}(t)-\mathbf{u}^{\infty}(t)\right\|_{L^{2}(U)} \underset{\sigma \rightarrow \infty}{\rightarrow 0}, \quad \text { for all } 0<t<T, \\
& \left\|\nabla \mathbf{u}^{\sigma}-\nabla \mathbf{u}^{\infty}\right\|_{L^{2}\left(0, T ; L^{2}(U)\right)} \underset{\sigma \rightarrow \infty}{\rightarrow 0},
\end{aligned}
$$

for any cylinder $U \subset \Omega$ of the form

$$
U=\left\{x=\left(x^{\prime}, x_{3}\right) \mid x^{\prime} \in D, x_{3} \in[0, \delta], \delta / \sigma \underset{\sigma \rightarrow \infty}{\longrightarrow} 0\right\} .
$$

On the other hand, $\left|x_{3}\right| / \sigma$ is $O(1)$ in $\Omega^{\sigma}$. Hence, it seems difficult to obtain any convergence estimate of $\mathbf{u}^{\sigma}$ to $\mathbf{u}^{\infty}$ globally in $\Omega^{\sigma}$ as $\sigma \rightarrow \infty$.

The previous result is not exactly what we aimed at, as it represents a way of approximating a general two-dimensional flow by a well-chosen helical flow. What we want, instead, is to show that helical flows with large $\sigma$ are nearly twodimensional. This adjustment is expressed in our next result.

Theorem 4.6. Fix $\sigma \geq 1$ and $T>0$. Let $\mathbf{u}_{0}^{\sigma} \in H_{0, p e r}^{1}\left(\Omega^{\sigma}\right)$ be a divergence-free, helical vector field. Let $\mathbf{u}^{\sigma}$ be the unique, strong helical solution of (2.7) on $[0, T$ ) with initial velocity $\mathbf{u}_{0}^{\sigma}$. There exists a (not necessarily unique) $\widetilde{\mathbf{w}_{0}^{\infty}} \in H^{1}(D)$, such that, if $\widetilde{\mathbf{u}^{\infty}}$ is the unique $2 \frac{1}{2} D$ solution of the Navier-Stokes equations (2.7) with initial data $\widetilde{\mathbf{u}_{0}^{\infty}}\left(\cdot, x_{3}\right)=\widetilde{\mathbf{w}^{\infty}} 0$, then for all $0<t<T$,

$$
\begin{aligned}
& \left\|\mathbf{u}^{\sigma}\left(t, \cdot, x_{3}=0\right)-\widetilde{\mathbf{u}^{\infty}}\left(t, \cdot, x_{3}=0\right)\right\|_{L^{2}(D)}+ \\
& \left\|\left.\nabla_{H} \mathbf{u}^{\sigma}\right|_{x_{3}=0}-\left.\nabla_{H} \widetilde{\mathbf{u}^{\infty}}\right|_{x_{3}=0}\right\|_{L^{2}\left(0, T ; L^{2}(D)\right)} \leq C(T) \frac{1}{\sqrt{\sigma}},
\end{aligned}
$$

where $C$ is independent of $\sigma \in[1, \infty)$.

We use the notation $\widetilde{\mathbf{u}^{\infty}}$ to emphasize that, while this is a solution of the limit problem, it is still dependent on $\sigma$ due to the correction to the initial condition to enforce the divergence-free condition.

Proof. As in the proof of Theorem 4.5, the traces of $\mathbf{u}^{\sigma}$ and $\mathbf{u}^{\infty}$ are well defined at the level of strong solutions. Furthermore, as in that theorem we will introduce a correction to the initial data $\mathbf{u}_{0}^{\sigma}$ to enforce the divergence-free condition on the initial data $\widetilde{\mathbf{u}^{\infty}}$ we take for the limit problem. Let $\mathbf{w}_{0}^{\sigma} \in H_{0}^{1}(D)$ be associated to the helical field $\mathbf{u}_{0}^{\sigma} \in H_{0, p e r}^{1}\left(\Omega^{\sigma}\right)$ by (2.8), satisfying (4.1). Let $\mathbf{w}^{\sigma}$ be the regular solution of (3.3) with this initial data. 
Next, let $\mathbf{v}_{0}^{\sigma}=\left(v_{0}^{\sigma, 1}, v_{0}^{\sigma, 2}\right) \in H_{0}^{1}(D)$ be a solution, given by Lemma 4.1, to the problem

$$
\operatorname{div}_{y} \mathbf{v}_{0}^{\sigma}=-\frac{2 \pi}{\sigma} E w_{0}^{\sigma, 3}
$$

where again $E$ is the differential operator defined in (2.4), such that

$$
\left\|\mathbf{v}_{0}^{\sigma}\right\|_{H^{1}} \leq C \frac{1}{\sigma}\left\|E w_{0}^{\sigma, 3}\right\|_{L^{2}(D)} \leq \frac{C}{\sigma}\left\|\mathbf{w}_{0}^{\sigma}\right\|_{H^{1}(D)} .
$$

Its existence is justified exactly as before.

We then set

$$
\widetilde{\mathbf{u}_{0}^{\infty}}(x)=\widetilde{\mathbf{w}^{\infty}}{ }_{0}\left(x^{\prime}\right):=\mathbf{w}_{0}^{\sigma}\left(x^{\prime}\right)-\left(\mathbf{v}_{0}^{\sigma}\left(x^{\prime}\right), 0\right),
$$

which is divergence free by 4.36). Let $\widetilde{\mathrm{w}^{\infty}}$ be the solution of 4.11 with initial data $\widetilde{\mathrm{w}^{\infty}}{ }_{0}$. The $2 \frac{1}{2} \mathrm{D}$ solution of the Navier-Stokes equations is given by $\widetilde{\mathbf{u}^{\infty}}(t, x)=\widetilde{\mathbf{w}^{\infty}}\left(t, x^{\prime}\right)$. In particular, the trace $\widetilde{\mathbf{u}^{\infty}}\left(t, \cdot, x_{3}=0\right)=\widetilde{w^{\infty}}(t, \cdot)$.

By Proposition 4.4 estimate (4.35) now follows from

$$
\begin{array}{r}
\left\|\mathbf{w}_{0}^{\sigma}-\widetilde{\mathbf{w}_{0}^{\infty}}\right\|_{L^{2}(D)}=\left\|\mathbf{v}_{0}^{\sigma}\right\|_{L^{2}(D)} \leq\left\|\mathbf{v}_{0}^{\sigma}\right\|_{H^{1}(D)} \leq \frac{C}{\sigma}\left\|\mathbf{w}_{0}^{\sigma}\right\|_{H^{1}(D)}, \\
\left\|\widetilde{\mathbf{w}_{0}^{\infty}}\right\|_{L^{2}(D)} \leq\left\|\mathbf{w}_{0}^{\sigma}\right\|_{L^{2}(D)}+\left\|\mathbf{v}_{0}^{\sigma}\right\|_{L^{2}(D)} \leq\left(1+\frac{C}{\sigma}\right)\left\|\mathbf{w}_{0}^{\sigma}\right\|_{H^{1}(D)}, \\
\left\|\mathbf{w}_{0}^{\sigma}\right\|_{L^{2}(D)} \leq\left\|\mathbf{w}_{0}^{\sigma}\right\|_{H^{1}(D)}=\left\|\mathbf{u}_{0}^{\sigma}\left(x_{3}=0\right)\right\|_{H^{1}(D)},
\end{array}
$$

with constants uniform in $\sigma \in[1, \infty)$.

\section{THE INVISCID CASE}

In this section we discuss symmetry reduction and the limit $\sigma \rightarrow \infty$ for the Euler equations under an additional geometric assumption, considered already in [5,6]. This assumption can be viewed as the analog of the no-swirl condition in axisymmetric flows and for this reason we will call it the no helical swirl or no helical stretching condition. It can be shown that the flow induced by solutions of the Euler equations preserves this condition at least when the solution is regular enough. Furthermore, vorticity has an especially simple form, being determined by its vertical component, which is advected by the flow. This observation allows to prove global existence and uniqueness of weak, helical solutions in much the same spirit as for solutions to the two-dimensional Euler equations, provided the initial velocity is bounded (cf. [9].)

We now briefly review these results, referring the reader to [5, 6] for more details. We will then discuss the limit problem as $\sigma \rightarrow \infty$ and converge of solutions. On one hand the limit problem is simpler, being given by the 2D Euler equations. In fact, under the no-stretching constraint the symmetry-reduced helical Euler equations becomes a two-dimensional systems for two components of the velocity, which admits a vorticity-stream function formulation (see e.g. [13].) This system is the analog of the symmetry-reduced equations 3.3 ) for the NavierStokes. On the other hand, to circumvent the lack of smoothing in the equations for 
positive time we will use compactness arguments to pass to the limit in $\sigma$, which do not provide a rate of convergence.

For ease of notation, we temporarily suppress the explicit $\sigma$-dependence of solutions and write $\mathbf{u}$ for $\mathbf{u}^{\sigma}$ for example. We assume for now that $\mathbf{u}$ and $p$ are smooth, so that all the manipulations to follow are justified.

Given that smooth, helical vector fields and functions are $\sigma$ periodic by Proposition 2.1. we state the initial-boundary-value problem for the Euler equations in the fundamental domain $\Omega^{\sigma}$ :

$$
\begin{cases}\partial_{t} \mathbf{u}+(\mathbf{u} \cdot \nabla) \mathbf{u}=-\nabla p, & \text { in }(0,+\infty) \times \Omega^{\sigma} ; \\ \operatorname{div} \mathbf{u}=0, & \text { in }[0,+\infty) \times \Omega^{\sigma} ; \\ \mathbf{u}\left(t, x^{\prime}, x_{3}\right) \cdot x^{\prime}=0, & \text { for } t \in[0,+\infty), \quad\left|x^{\prime}\right|=1,0 \leq x_{3} \leq \sigma ; \\ \mathbf{u}\left(t, x^{\prime}, 0\right)=\mathbf{u}\left(t, x^{\prime}, \sigma\right) & \text { for } t \in[0,+\infty), \quad x^{\prime} \in D ; \\ p\left(t, x^{\prime}, 0\right)=p\left(t, x^{\prime}, \sigma\right) & \text { for } t \in[0,+\infty), \quad x^{\prime} \in D ; \\ \mathbf{u}(0, x)=\mathbf{u}_{0}, & x \in \Omega^{\sigma},\end{cases}
$$

where again $x=\left(x^{\prime}, x_{3}\right)$ and $x^{\prime}=\left(x_{1}, x_{2}\right)$.

Let

$$
\boldsymbol{\xi}:=\left(x_{2},-x_{1}, \frac{\sigma}{2 \pi}\right)=-\mathbf{x}_{H}^{\perp}+\frac{\sigma}{2 \pi} \mathbf{e}_{3} .
$$

We will consider flows satisfying the following no-helical-swirl or stretching condition:

$$
\mathbf{u} \cdot \boldsymbol{\xi}=0
$$

This condition is preserved by smooth flows under the time evolution governed by the Euler equations.

There are several consequences of this condition. Firstly, the vertical component $u_{3}$ of the velocity field $\mathbf{u}$ is computed from the other two components, i.e., the dynamics is planar. Secondly, the vorticity $\boldsymbol{\omega}=\operatorname{curl}_{x} \mathbf{u}$ is given by

$$
\boldsymbol{\omega}(t, x)=\frac{2 \pi}{\sigma} \omega(t, x) \boldsymbol{\xi}, \quad \omega:=\omega^{3},
$$

where $\omega^{3}$ is the component of the vorticity along the axis of the cylinder $\Omega$. Furthermore, $\omega$ is advected by the flow $\mathbf{u}$ :

$$
\partial_{t} \omega+\mathbf{u} \cdot \nabla \omega=0 .
$$

To derive the symmetry-reduced equations, we recall that $\mathbf{w}(t, y)=\mathbf{u}(t, y, 0)$ from Proposition 2.1, given that the matrices $M$ and $m$ becomes the identity matrix for $x_{3}=0$. Consequently,

$$
\varpi(t, y):=\omega(t, y, 0)=-\nabla_{y}^{\perp} \mathbf{w}_{H}(t, y)=\operatorname{curl}_{y} \mathbf{w}_{H}(t, y) .
$$

Above, to avoid introducing further notation, we have abused notation slightly and identified $\left(w^{1}, w^{2}\right)$ with $\mathbf{w}_{H}=\left(w^{1}, w^{2}, 0\right)$, where $w^{1}, w^{2}$ are the horizontal components of $\mathbf{w}$ with respect to the standard Cartesian frame in $\mathbb{R}^{3}$.

While $\mathbf{w}_{H}$ is not divergence free in view of $(3.3 \mathrm{~b}$ ), one observes that a divergencefree 2D flow can be constructed from $w$ under the no-helical-swirl condition, which 
therefore admits a stream function $\psi$ on $D$. This stream function satisfies:

$$
\left\{\begin{array}{l}
\partial_{y_{1}} \psi=\frac{4 \pi^{2}}{\sigma^{2}}\left[-y_{1} y_{2} w^{1}+\left(\frac{\sigma^{2}}{4 \pi^{2}}+y_{1}^{2}\right) w^{2}\right], \\
\partial_{y_{2}} \psi=-\frac{4 \pi^{2}}{\sigma^{2}}\left[\left(\frac{\sigma^{2}}{4 \pi^{2}}+y_{2}^{2}\right) w^{1}-y_{1} y_{2} w^{2}\right] .
\end{array}\right.
$$

We define the following matrix:

$$
H(y):=\frac{4 \pi^{2}}{\sigma^{2}}\left[\begin{array}{cc}
\left(\frac{\sigma^{2}}{4 \pi^{2}}+y_{2}^{2}\right) & -y_{1} y_{2} \\
-y_{1} y_{2} & \left(\frac{\sigma^{2}}{4 \pi^{2}}+y_{1}^{2}\right)
\end{array}\right],
$$

and rewrite as

$$
\nabla_{y}^{\perp} \psi=H(y) \mathbf{w}_{H} .
$$

A direct calculation, as in [6], then shows that

$$
\begin{aligned}
\operatorname{curl}_{H} & =\operatorname{div} K(y) \nabla_{y} \psi, \quad \text { with } \\
K(y) & :=\frac{1}{\frac{\sigma^{2}}{4 \pi^{2}}+|y|^{2}}\left[\begin{array}{cc}
\left(\frac{\sigma^{2}}{4 \pi^{2}}+y_{1}^{2}\right) & y_{1} y_{2} \\
y_{1} y_{2} & \left(\frac{\sigma^{2}}{4 \pi^{2}}+y_{2}^{2}\right)
\end{array}\right] .
\end{aligned}
$$

From (5.6) and (5.9), it follows that

$$
\varpi=\mathcal{L}_{H} \psi,
$$

where the operator $\mathcal{L}_{H}$ is defined by:

$$
\mathcal{L}_{H}:=\operatorname{div}_{y}\left(K(y) \nabla_{y}\right) .
$$

It is not difficult to show that $\mathcal{L}_{H}$ is a second-order, scalar, strongly elliptic operator. Consequently, $\nabla^{2} \mathcal{L}_{H}$ is a singular integral.

Next, calculus inequalities show that the transport equation (5.5) for $\omega$ reduces by helical symmetry (i.e., using the correspondence in Proposition 2.1) to the following equation for $\varpi$ on $(0, T) \times D$ :

$$
\partial_{t} \varpi+\mathbf{w}_{H} \cdot \nabla_{y} \varpi+\frac{4 \pi^{2}}{\sigma^{2}}\left(y^{\perp} \cdot \mathbf{w}_{H}\right) E \varpi=0,
$$

where $E$ is again the operator given in (2.4). Using (5.9), we can rewrite this equation as an equation for $\varpi$ and $\psi$ only (cf. [6, Lemma 2.17].) Furthermore, we can choose Dirichlet boundary conditions for $\psi$ from the no-penetration condition for $\mathbf{u}$ as in Corollary 2.16 of [6]. Therefore, under the no-helical swirl condition and for sufficiently regular solutions, the initial-boundary-value (5.1) for the Euler equations is equivalent to the following symmetry-reduced system:

$$
\begin{array}{lr}
\partial_{t} \varpi+\partial_{y_{1}} \psi \partial_{y_{2}} \varpi-\partial_{y_{2}} \psi \partial_{y_{1}} \varpi=0, & y \in D, 0<t<T, \\
\varpi=\mathcal{L}_{H} \psi, & y \in D, 0<t<T, \\
\psi(0, y)=\psi_{0}(y), & y \in D, \\
\left.\psi\right|_{\partial D}=0, & y \in D .
\end{array}
$$

Since $5.11 \mathrm{a}$ ) is a transport equation by the divergence-free vector field $\nabla_{y}^{\perp} \psi$, the $L^{\infty}$ norm of the reduced vorticity $\varpi$ is preserved under the flow. By (5.4) and Proposition 2.1 the vorticity $\boldsymbol{\omega}=\operatorname{curl}_{x} \mathbf{u}$ is preserved under the flow induced by 
u. By the Beale-Kato-Maja criterion ( see e.g. [13]) then, smooth helical solutions of (5.1) are global in time and agree with weak solutions with the same initial data.

We next discuss weak solution. Given $\psi_{0} \in H_{0}^{1}(D) \cap H^{2}(D)$, we call a function $\psi \in L^{1}\left([0, T) ; H_{0}^{1}(D) \cap H^{2}(D)\right)$ a weak solution of the above system on $[0, T)$ with initial data $\psi_{0}$ if, for all test function $\phi \in C_{c}^{\infty}([0, T) \times D), \psi$ satisfies:

$$
\begin{array}{r}
\int_{D} \mathcal{L}_{H} \psi_{0} \phi(0) d y-\int_{0}^{T} \int_{D} \mathcal{L}_{H} \psi \partial_{t} \phi d y d t+\int_{0}^{T} \int_{D} \partial_{y_{2}} \psi \mathcal{L}_{H} \psi \partial_{y_{1}} \phi d y d t \\
-\int_{0}^{T} \int_{D} \partial_{y_{1}} \mathcal{L}_{H} \psi \partial_{y_{2}} \phi d y d t=0 .
\end{array}
$$

Ettinger and Titi [6] proved that there exists a unique weak solution on $[0, T)$, for all $T>0$, provided in addition $\mathcal{L}_{H} \psi_{0} \in L^{\infty}(D)$. In this case the solution satisfies $\mathcal{L}_{H} \psi \in L^{\infty}((0, T) \times D)$.

While there is an existence theory for weak solutions of the Euler equation in three dimensions [4, 17], we will give here a definition of weak solution to (5.1) adapted to the geometry of the problem and amenable to the analysis of the limit $\sigma \rightarrow \infty$ (for further discussion on the uniqueness of helical weak solutions, we refer the reader to [1].) Let $\psi$ be the unique weak solution of (5.11) with initial condition $\psi_{0} \in H_{0}^{1}(D) \cap H^{2}(D)$ such that $\mathcal{L}_{H} \psi_{0} \in L^{\infty}(D)$. Let $\mathbf{w}=\left(\mathbf{w}_{H}, w^{3}\right)$, where $\mathbf{w}_{H}$ is given in (5.9) and $w^{3}$ is obtained from $\mathbf{w}_{H}$ via (5.3) as

$$
w^{3}=\frac{2 \pi}{\sigma} y^{\perp} \cdot \mathbf{w}_{H}
$$

Let $\mathbf{u}$ be defined from $\mathbf{w}$ by (2.8). We will call $\mathbf{u}$ a weak, helical solution of (5.1). This definition is justified in view of the following proposition.

Proposition 5.1. Let $\left\{\psi_{0, n}\right\}$ be a sequence of functions converging to $\psi_{0} \in H_{0}^{1}(D) \cap$ $H^{2}(D)$. Let $\psi_{n}$ be the smooth solution of (5.11a) with initial data $\psi_{0, n}$. Then, $\psi_{n}$ converges uniformly on $[0, T) \times D$ ) to $\psi$ the unique weak solution of (5.11).

The proof is contained in [6]. We recall it briefly.

Proof. The sequence $\left\{\psi_{n}\right\}$ is uniformly bounded in $L^{1}\left([0, T) ; H_{0}^{1}(D) \cap H^{2}(D)\right)$ and $\mathcal{L}_{H} \psi$ is uniformly bounded in $L^{\infty}([0, T) \times D)$. Recall that the equation for $\varpi_{n}=\mathcal{L}_{H} \psi_{n}$ is a transport equation by $\nabla_{y}^{\perp} \psi_{n}$, which is divergence free. Since $\partial_{i} \partial_{j} \mathcal{L}_{H}$ is a Calderon-Zygmund singular integral, $\left\{\nabla_{y}^{\perp} \psi_{n}\right\}$ is bounded in the space LLip of Log-Lipschitz vector fields. Hence, the family $\left\{X_{n}\right\}$, where $X_{n}$ is the flow generated by $\nabla^{\perp} \psi_{n}$ is equicontinuous and hence, upon possibly passing to subsequences, $\varpi_{n}$ converges strongly in $L^{1}((0, T) \times D)$ and $\nabla_{y}^{\perp} \psi_{n}$ converges uniformly to $\nabla_{y}^{\perp} \psi_{n}$. In particular, $\psi_{n}$ converges uniformly to $\psi$. These convergence results are enough to pass to the limit in the weak formulation (5.12) (cf. [13, Section 8.2.2].) The $\operatorname{limit}_{n \rightarrow \infty} \lim _{n}$ must necessarily agree with $\psi$ by uniqueness of the solution, so the whole sequence converges to $\psi$.

This result also implies that, if $\varpi(0) \in L^{\infty}(D)$, then $\varpi(t, x)=\varpi\left(X^{-1}(t, x)\right.$, where $X$ is the flow generated by $\nabla^{\perp} \psi$, is the (unique) weak solution of (5.11), hence all its $L^{p}$ norms are constant in time. 
We next discuss the limit $\sigma \rightarrow \infty$. We reinstate the explicit dependence on $\sigma$, and write for example $\mathbf{u}^{\sigma}$ for the solution of (5.1), $\boldsymbol{\omega}^{\sigma}$ for $\operatorname{curl}_{x} \mathbf{u}^{\sigma}$ and so on. We denote the corresponding quantities in the limit by $\mathbf{u}^{\infty}, \boldsymbol{\omega}^{\infty}$ and so on.

Formally taking the limit $\sigma \rightarrow \infty$ in (5.3) gives $u^{\infty, 3} \equiv 0$ and, hence, $\mathbf{u}^{\infty}=$ $\mathbf{u}_{H}^{\infty}$. Furthermore, $\mathbf{u}^{\infty}$ becomes independent of the $x_{3}$ variable, so that

$$
\mathbf{u}^{\infty}(x)=\mathbf{w}^{\infty}\left(x^{\prime}\right)=\mathbf{w}_{H}^{\infty}\left(x^{\prime}\right)
$$

is divergence-free as a vector field on $D$. Also, the matrix $K^{\sigma}$ approaches the identity matrix in the limit, so that $\mathcal{L}_{H}^{\infty}$ is simply the Laplace operator, $\psi^{\infty}$ is the stream functions associated to $\mathbf{u}_{H}^{\infty}$, and $\varpi^{\infty}\left(x^{\prime}\right)=\omega^{\infty}(x)=\operatorname{curl}_{x^{\prime}} \mathbf{u}_{H}^{\infty}(x)$. In particular, 5.11a becomes the vorticity-stream function formulation of the 2D Euler equations. We conclude that, at least formally, helical solutions to the 3D Euler equations become planar 2D solutions of the Euler equations as $\sigma \rightarrow \infty$.

We explicitly state the limit problem:

$$
\begin{array}{lr}
\partial_{t} \varpi^{\infty}+\partial_{y_{1}} \psi^{\infty} \partial_{y_{2}} \varpi^{\infty}-\partial_{y_{2}} \psi^{\infty} \partial_{y_{1}} \varpi^{\infty}=0, & y \in D, 0<t<T, \\
\varpi^{\infty}=\Delta_{y} \psi^{\infty}, & y \in D, 0<t<T, \\
\psi^{\infty}(0, y)=\psi_{0}^{\infty}(y), & y \in D, \\
\left.\psi^{\infty}\right|_{\partial D}=0, & y \in D .
\end{array}
$$

Below we will study convergence of the corresponding stream functions $\psi^{\sigma} \rightarrow$ $\psi^{\infty}$ as $\sigma \rightarrow \infty$. Since the uniqueness and regularity of weak solutions depends on an $L^{\infty}$ control on the vorticity, we will prescribe the initial vorticity $\varpi_{0}^{\sigma}$ independent of $\sigma$, i.e.,

$$
\varpi_{0}^{\infty}=\varpi_{0}^{\sigma}=\varpi_{0} \in L^{\infty}(D)
$$

This choice $\mathrm{n}$ can be relaxed by taking a sequence $\varpi_{0}^{\sigma}$ converging to $\varpi_{0}$ strongly in $L^{\infty}(D)$. We then obtain an initial condition for the stream function, $\psi_{0}^{\sigma}$, that is $\sigma$-dependent. We choose the initial data for the stream function as the unique solution in $H_{0}^{1}(D)$ of the following problems, respectively:

$$
\begin{aligned}
\Delta \psi_{0}^{\infty} & =\varpi_{0}, \\
\mathcal{L}_{H}^{\sigma} \psi_{0}^{\sigma} & =\varpi_{0} .
\end{aligned}
$$

By elliptic regularity, $\psi_{0}^{\infty}, \psi_{0}^{\sigma} \in W^{2, p}$ for all $1<p<\infty$.

Next we will derive uniform bounds in $\sigma$ on the $W^{2, p}$ norm of $\psi^{\sigma}$ and then use compactness arguments to pass to the limit. It is well known that, under the condition that the initial vorticity $\varpi_{0}$ is bounded, solutions to the 2D Euler equations are global in time and unique [9]. Therefore, it will be enough to establish convergence along subsequences.

Lemma 5.2. Let $1<p<\infty$ be fixed. Then, there exists a constant $C_{p}>0$ such that, for all $\sigma>1$ and for all $f \in W^{2, p}(D)$,

$$
\left\|\mathcal{L}_{H} f\right\|_{L^{p}(D)} \leq C_{p}\|f\|_{W^{2, p}(D)} .
$$

Moreover, there exists $a \sigma_{0}>1$ and a constant $C_{p}>0$, independent of $\sigma \in$ $\left[\sigma_{0}, \infty\right)$ such that

$$
\|f\|_{W^{2, p}(D)} \leq C_{p}\left\|\mathcal{L}_{H} f\right\|_{L^{p}(D)} .
$$


Proof. We observe that we can write the matrix $K^{\sigma}=I_{2}+F^{\sigma}$, where $I_{2}$ is the $2 \times 2$-identity matrix and

$$
F^{\sigma}(y)=\frac{1}{1+\frac{4 \pi^{2}|y|^{2}}{\sigma^{2}}}\left[\begin{array}{cc}
\frac{4 \pi^{2} y_{1}^{2}}{\sigma^{2}} & \frac{4 \pi^{2} y_{1} y_{2}}{\sigma^{2}} \\
\frac{4 \pi^{2} y_{1} y_{2}}{\sigma^{2}} & \frac{4 \pi^{2} y_{2}^{2}}{\sigma^{2}}
\end{array}\right] .
$$

We have:

$$
\left\|F^{\sigma}\right\|_{L^{\infty}(D)} \leq C_{1} \frac{1}{\sigma^{2}}, \quad\left\|\nabla_{y} F^{\sigma}\right\|_{L^{\infty}(D)} \leq C_{2} \frac{1}{\sigma^{2}},
$$

for some constants $C_{1}, C_{2}$ independent of $\sigma$. The bound (5.15) then follows immediately.

To establish (5.16), we write

$$
\Delta_{y} f=\mathcal{L}_{H} f-F^{\sigma}(y): \nabla^{2} f-\left(\operatorname{div}_{y} F^{\sigma}(y)\right) \cdot \nabla_{y} f
$$

so that from elliptic regularity for the Poisson's problem for $1<p<\infty$, Hölder's inequality and (5.17):

$$
\begin{aligned}
\|f\|_{W^{2, p}} & \leq C_{p}^{\prime}\left\|\mathcal{L}_{H} f\right\|_{L^{p}}+\left\|F^{\sigma}: \nabla^{2} f\right\|_{L^{p}}+\left\|\operatorname{div}_{y} F^{\sigma} \cdot \nabla_{y} f\right\|_{L^{p}} \\
& \leq C_{p}^{\prime}\left\|\mathcal{L}_{H} f\right\|_{L^{p}}+C_{1} \frac{1}{\sigma^{2}}\left\|\nabla_{y}^{2} f\right\|_{L^{p}}+C_{2} \frac{1}{\sigma^{2}}\left\|\nabla_{y} f\right\|_{L^{p}},
\end{aligned}
$$

or equivalently:

$$
\left(1-\left(C_{1}+C_{2}\right) / \sigma^{2}\right)\|f\|_{W^{2, p}} \leq C_{p}^{\prime}\left\|\mathcal{L}_{H} f\right\|_{L^{p}}
$$

So, the result follows provided we choose $\sigma_{0}>1 / \sqrt{\left(C_{1}+C_{2}\right)}$.

We now state and prove our convergence result for the Euler equations. We recall that the only difference between the equations at $\sigma$ finite and in the limit is the equation expressing the relationship between the vorticity and the stream function.

Theorem 5.3. Let $\varpi_{0} \in L^{\infty}(D)$. Let $\psi_{0}^{\sigma}$ and $\psi_{0}^{\infty}$ be given by (5.14). Let $\psi^{\sigma}$ be the unique weak solution of (5.11) with initial data $\psi_{0}^{\sigma}$. Let $\psi^{\infty}$ be the unique weak solution of (5.13) with initial data $\psi_{0}^{\infty}$. Then, $\psi^{\sigma}$ converges to $\psi$ weakly in $L^{p}\left([0, T) ; W^{1, p}(D)\right)$.

Proof. Since the initial vorticity $\varpi_{0} \in L^{\infty}(D), \nabla^{\perp} \psi^{\sigma} \in \operatorname{LLip}(D)$ with a bound on the Log-Lipschitz norm that is uniform in $\sigma$ for $\sigma \in[1, \infty)$ by (5.17). Therefore, we have a uniform bound on $\varpi^{\sigma}$ in $L^{\infty}([0, T) \times D)$, thanks to the transport equation (5.11a). In turn by (5.16), this bound implies a bound on the family $\left\{\psi^{\sigma}\right\}$ of weak solutions of (5.11) in all spaces $L^{\infty}\left([0, T) ; W^{2, p}\right), 1<p<\infty$ that is uniform in $\sigma \geq \sigma_{0}$ for $\sigma_{0}$ large enough.

Next, we recall the following a priori bound for weak solutions of [5.11) (se [6, Lemma 4.2]):

$$
\left\|\partial_{t} \psi^{\sigma}\right\|_{L^{\infty}\left([0, T) ; W^{1, p}(D)\right)} \leq C_{p}\left\|\mathcal{L}_{H}^{\sigma} \psi^{\sigma}\right\|_{L^{\infty}((0, T) \times D)}\left\|\psi^{\sigma}\right\|_{L^{\infty}\left([0, T) ; W^{1, p}\right)},
$$

where $C_{p}$ is independent of $\sigma$ for $\sigma$ large enough as in Lemma 5.2 Therefore, $\left\{\psi^{\sigma}\right\}$ is uniformly bounded in $\operatorname{Lip}\left([0, T) ; W^{1, p}(D)\right)$. By the Aubin compactness theorem (see e.g. [3, Lemma 8.4]) then, there is a sequence $\left\{\psi^{\sigma_{n}}\right\}$ that converges 
strongly in $L^{\infty}\left([0, T) ; W^{1, p}\right)$ to a function $\psi^{\infty}$. Upon passing to a subsequence if necessary, one can assume also that $\varpi^{\sigma_{n}}$ converges weakly-* in $L^{\infty}([0, T) \times D)$ to a function $\varpi^{\infty}$ from the uniform bound obtained above. It remains to show that $\varpi^{\infty}=\Delta \psi^{\infty}$ in $L^{2}(D)$. This result follows from the identity $\varpi^{\sigma}=\mathcal{L}_{H} \psi^{\sigma}$, valid for all $\sigma$, and (5.17), by writing again $K^{\sigma}=I_{2}+F^{\sigma}$.

As in the proof of Proposition 5.8 in [6], these convergence results are sufficient to show that $\psi^{\infty}$ and $\varpi^{\infty}$ satisfy the weak formulation of the limit problem (5.13). But weak solutions of the 2D Euler equations are unique if the vorticity is bounded, hence any converging sequence of $\left\{\psi^{\sigma}\right\}$ must converge to $\psi^{\infty}$.

\section{ACKNOWLEDGMENTS}

M. L. F. and H. N. L. wish to thank the University of California at Riverside, where part of this work was conducted, for their hospitality. M. L. F. is partially supported by Brazil CNPq grant 303089/2010-5, and CNPq fellowship 200434/20110. H. N. L. is partially supported by Brazil CNPq grant 306331/2010-1, CAPES fellowship 6649/10-6, and FAPERJ grant E-26/103.197/2012. A. M. would like to thank the Institute of Mathematics at the Federal University in Rio de Janeiro for their hospitality and support. A. M.'s work is partially supported by the US National Science Foundation grants DMS-1009713 and DMS-1009714. D. N.'s work is partially supported by the Chinese National Youth grant 11001184. The work of E. S. T. was supported in part by the Minerva Stiftung/Foundation, and the National Science Foundation grants DMS-1009950, DMS-1109640 and DMS-1109645.

\section{REFERENCES}

1. C. Bardos, M. C. Lopes Filho, D. Niu, H. J. Nussenzveig Lopes, and E. S. Titi, Stability of twodimensional viscous incompressible flows under three-dimensional perturbations and inviscid symmetry breaking, to appear in SIAM J. Math. Anal., ArXiv Preprint 1201.2742v2.

2. S. Childress, M. Landman, and H. Strauss, Steady motion with helical symmetry at large Reynolds number, Topological fluid mechanics (Cambridge, 1989), Cambridge Univ. Press, Cambridge, 1990, pp. 216-224. MR 1093923

3. P. Constantin and C. Foias, Navier-Stokes equations, Chicago Lectures in Mathematics, University of Chicago Press, Chicago, IL, 1988. MR 972259 (90b:35190)

4. C. De Lellis and L. Székelyhidi, Jr., The Euler equations as a differential inclusion, Ann. of Math. (2) 170 (2009), no. 3, 1417-1436. MR 2600877 (2011e:35287)

5. A. Dutrifoy, Existence globale en temps de solutions hélicoïdales des équations d'Euler, C. R. Acad. Sci. Paris Sér. I Math. 329 (1999), no. 7, 653-656. MR 1717127 (2000f:76011)

6. B. Ettinger and E. S. Titi, Global existence and uniqueness of weak solutions of threedimensional Euler equations with helical symmetry in the absence of vorticity stretching, SIAM J. Math. Anal. 41 (2009), no. 1, 269-296. MR 2505860 (2011c:35445)

7. G. P. Galdi, An introduction to the mathematical theory of the Navier-Stokes equations. Vol. I, Springer Tracts in Natural Philosophy, vol. 38, Springer-Verlag, New York, 1994, Linearized steady problems. MR 1284205 (95i:35216a)

8. D. Iftimie and G. Raugel, Some results on the Navier-Stokes equations in thin 3D domains, J. Differential Equations 169 (2001), no. 2, 281-331, Special issue in celebration of Jack K. Hale's 70th birthday, Part 4 (Atlanta, GA/Lisbon, 1998). MR 1808469 (2001m:35254)

9. V. I. Judovič, Non-stationary flows of an ideal incompressible fluid, Z̆. Vyčisl. Mat. i Mat. Fiz. 3 (1963), 1032-1066. MR 0158189 (28 \#1415) 
10. O. A. Ladyzhenskaya, The mathematical theory of viscous incompressible flow, Second English edition, revised and enlarged. Translated from the Russian by Richard A. Silverman and John Chu. Mathematics and its Applications, Vol. 2, Gordon and Breach Science Publishers, New York, 1969. MR 0254401 (40 \#7610)

11. E. H. Lieb and M. Loss, Analysis, second ed., Graduate Studies in Mathematics, vol. 14, American Mathematical Society, Providence, RI, 2001. MR 1817225 (2001i:00001)

12. A. Mahalov, E. S. Titi, and S. Leibovich, Invariant helical subspaces for the Navier-Stokes equations, Arch. Rational Mech. Anal. 112 (1990), no. 3, 193-222. MR 1076072 (91h:35252)

13. A. J. Majda and A. L. Bertozzi, Vorticity and incompressible flow, Cambridge Texts in Applied Mathematics, vol. 27, Cambridge University Press, Cambridge, 2002. MR 1867882 (2003a:76002)

14. H. Sohr, The Navier-Stokes equations, Birkhäuser Advanced Texts: Basler Lehrbücher. [Birkhäuser Advanced Texts: Basel Textbooks], Birkhäuser Verlag, Basel, 2001, An elementary functional analytic approach. MR 1928881 (2004b:35265)

15. M. E. Taylor, Partial differential equations III. Nonlinear equations, second ed., Applied Mathematical Sciences, vol. 117, Springer, New York, 2011. MR 2744149 (2011m:35003)

16. R. Temam, Navier-Stokes equations, AMS Chelsea Publishing, Providence, RI, 2001, Theory and numerical analysis, Reprint of the 1984 edition. MR 1846644 (2002j:76001)

17. E. Wiedemann, Existence of weak solutions for the incompressible Euler equations, Ann. Inst. H. Poincaré Anal. Non Linéaire 28 (2011), no. 5, 727-730. MR 2838398 (2012h:35270)

(M.C. Lopes Filho) Mathematics Institute,, Federal University of Rio de Janeiro,, P.O. BOX 68530, 21941-909 RIO DE JANEIRO, RJ, BRAZIL

E-mail address: mlopes@im.ufrj.br

(A.L. Mazzucato) Department of Mathematics, Penn State University, University PARK, PA, 16801, U.S.A.

E-mail address: alm24 epsu. edu

(D. Niu) School of Mathematical Sciences, Capital Normal University, Beijing 100048, P. R. CHINA

E-mail address: niuniudjegmail.com

(H.J. Nussenzveig Lopes) Mathematics Institute, Federal University of Rio de JANEIRO,, P.O. BoX 68530, 21941-909 Rio DE JANEIRO, RJ, BRAZIL

E-mail address: hlopes@im.ufrj.br

(E.S. Titi) Department of Computer Science And Applied Mathematics, Weizmann Institute of Science, Rehovot 76100, Israel, And, Department of Mathematics, and Department of Mechanical and Aerospace Engineering, University of CaliFORNIA, IRVINE, CA 92697-3875, USA.

E-mail address: etiti@math.uci.edu, edriss.titi@weizmann.ac.il 